\title{
Perirhinal-amygdala circuit-level computational model of temporal encoding in fear conditioning
}

\author{
KINH H. TIEU, ANDREW L. KEIDEL, JOHN P. MCGANN, \\ BILLIE FAULKNER, and THOMAS H. BROWN \\ Yale University, New Haven, Connecticut
}

\begin{abstract}
Here we present a real-time model of fear conditioning in which the functional anatomy and neurophysiology of the lateral amygdala and perirhinal cortex provide a mechanism for temporal learning during Pavlovian conditioning. The model uses realistic neuronal and circuit dynamics to map time onto space and relies on a conventional Hebbian learning rule that requires strict temporal contiguity for synaptic modification. The input-output relationships of the model neurons simulate our physiological recordings with respect to latency to fire, firing frequency, and accommodation tendency. Chains of these neurons form a spectrum of activity windows delayed by various amounts from the conditioned stimulus onset. Simulations reveal that learning occurs only when the conditioned and unconditioned stimuli are explicitly paired, that the interstimulus interval (ISI) is accurately learned over a time range from 0.5 to $16 \mathrm{sec}$, and that low-frequency noise causes the accuracy of temporal learning to decrease as the ISI increases, in accordance with a Weber-type law.
\end{abstract}

Pavlovian conditioning furnishes a productive tool and framework for exploring the neurobiology of learning and memory in mammals (Fanselow, 1998; Gormezano, Prokasy, \& Thompson, 1987; Kehoe, 1990; Kim, Krupa, \& Thompson, 1998; Kim \& Thompson, 1997; Lam, Wong, Canli, \& Brown, 1996; Lavond, Kim, \& Thompson, 1993; LeDoux, 1995; Mauk \& Donegan, 1997; Moore \& Choi, 1997; Schmajuk, 1997; Thompson, 1986; Thompson \& Krupa, 1994). In a typical delay conditioning paradigm, the conditioned stimulus (CS) onset precedes the unconditioned stimulus (US) onset, and the CS and the US co-terminate. Under these circumstances, the animal not only acquires a conditioned response (CR) to the CS, but also learns about the CS--US interstimulus time interval (ISI). Temporal learning is evident from the fact that the CR is timed to peak near the US onset.

Temporal learning is increasingly recognized as fundamental to understanding mechanisms of conditioning, emotion, cognition, and motor control (Bouton, 1993; Churchland, 1995; Gibbon, Malapani, Dale, \& Gallistel, 1997; Harrington \& Haaland, 1998; Ivry, 1996; Kesner, 1992; Mauk \& Donegan, 1997; Moore, Choi, \& Brun-

This research was supported by grants from the Office of Naval Research to T.H.B., the National Institutes of Health to T.H.B., and the National Institute of Aging to B.F., as well as by a National Science Foundation graduate fellowship to B.F. We thank Angela Szeto for assistance with figure preparation and the reviewers for valuable suggestions. K.H.T. and A.L.K. are in the Department of Computer Science. J.P.M. is in the Department of Psychology and the Interdepartmental Neuroscience Program. B.F. is in the Interdepartmental Neuroscience Program. T.H.B. is in the Department of Psychology, the Department of Cellular and Molecular Physiology, and the Interdepartmental Neuroscience Program. Correspondence concerning this article should be addressed to T. H. Brown, Yale University, P.O. Box 208205, New Haven, CT 06520-8205 (e-mail: thomas.brown@yale.edu). zell, 1998; Raymond, Lisberger, \& Mauk, 1996; Rosenbaum \& Collyer, 1998; Schmajuk, 1997). It has been suggested that time is the "primordial context" (Gibbon et al., 1997) and that cognitive systems form "temporal maps" that define the proximity in time of events in the world, enabling "temporal expectations of environmental events" (Schmajuk, 1997). There is a growing consensus that temporal aspects of behavior must be addressed in modern models of Pavlovian conditioning (Desmond, 1990; Grossberg \& Schmajuk, 1989; Kehoe, 1988, 1990; Kehoe, Horne, Macrae, \& Horne, 1993; Mauk \& Donegan, 1997; Moore, Berthier, \& Blazis, 1990; Moore \& Choi, 1997; Moore et al., 1998; Moore, Desmond, \& Berthier, 1989; Schmajuk, 1997; Sutton \& Barto, 1981, 1990), especially in those that seek to make contact with the underlying neurobiological mechanisms.

One natural and general approach to temporal information processing is to map time onto space (Jeffress, 1948). For very short time intervals $(0-\sim 650 \mu \mathrm{sec})$, the action potential propagation duration, combined with specialized coincidence detection circuits, can perform this mapping via a spectrum of delay lines (Carr \& Konishi, 1988). For somewhat longer time intervals $(0.5-500 \mathrm{msec}$ or even longer), the neurobiological encoding mechanisms are less obvious. Models have included time-varying activity vectors (Buonomano \& Mauk, 1994); activitydependent properties of synaptic transmission, such as facilitation and slow inhibition (Buonomano \& Merzenich, 1995); arrays of tap delay lines (Desmond \& Moore, 1988; Moore et al., 1990; Moore et al., 1989) or cascades of spreading activation (Moore \& Choi, 1997); groups of neurons oscillating at different frequencies (Church \& Broadbent, 1991; Gluck, Reifsnider, \& Thompson, 1990; Mial, 1989); and time-dependent stimulus traces (Schmajuk, 1997). Even greater durations $(0.5 \mathrm{sec}$ to tens of sec- 
onds) are especially relevant to studies of fear conditioning, where it seems that rats can learn relatively long CS-US intervals (J. S. Brown, Kalish, \& Farber, 1951; Davis, Schlesinger, \& Sorenson, 1989).

Models designed to accommodate these longer intervals commonly invoke notions of endogenous clocks (B. L. Brown, Hemmes, \& Cabeza de Vaca, 1992; Ivry, 1996; Treisman, Cook, Naish, \& MacCrone, 1994), an idea whose cellular basis remains uncertain. We have been examining the perirhinal cortex (PR) and the adjacent lateral amygdala (ALa) area-brain regions thought to be involved in fear conditioning (Cahill \& McGaugh, 1998; Corodimas \& LeDoux, 1995; Fanselow, 1998; Fanselow \& Kim, 1994; LeDoux, 1995; Maren, 1996) - for candidate neurobiological timing mechanisms (Faulkner, 1997; Faulkner \& Brown, 1995, 1996, in press). Of particular interest to the present investigation is the hypothesis that the amygdala is involved in encoding and processing the interaction between temporal information and affect, including "the time at which an affect-laden experience occurs" (Kesner, 1992).

At the synaptic level, a common assumption is that associative long-term synaptic potentiation (LTP; Barrionuevo \& Brown, 1983; Kelso \& Brown, 1986), a Hebbian form of synaptic plasticity (T. H. Brown, Kairiss, \& Keenan, 1990; Kelso, Ganong, \& Brown, 1986), may be a substrate for certain forms of Pavlovian conditioning (Chapman, Kairiss, Keenan, \& Brown, 1990; Kelso \& Brown, 1986; Lam et al., 1996; LeDoux, 1990, 1995; Maren, 1996). One conventional model of fear conditioning postulates that, during acquisition, the CS and US converge in the ALa (Lam et al., 1996; LeDoux, 1990, 1993; Le Gal La Salle \& Ben-Ari, 1981; Maren, 1996; Quirk, Repa, \& LeDoux, 1995; Romanski, Clugnet, Bordi, \& LeDoux, 1993) and that associative LTP can occur in cells that receive simultaneous input from both stimuli (Armony, Servan-Schreiber, Cohen, \& LeDoux, 1995; Bordi, LeDoux, Clugnet, \& Pavlides, 1993; LeDoux, 1990, 1993, 1995; LeDoux, Cicchetti, Xagoraris, \& Romanski, 1990; Maren, 1996; Quirk et al., 1995; Romanski et al., 1993). The essential idea is that associative LTP can increase the strength of the previously weak CS input (see T. H. Brown et al., 1990; Kelso \& Brown, 1986; Kelso et al., 1986) to the extent that the CS-driven connections become sufficiently strong to generate output from the amygdala (Kapp, Whalen, Supple, \& Pascoe, 1992; Lam et al., 1996; LeDoux, 1993, 1995; Maren, 1996). This CS-generated output from the amygdala can orchestrate changes in the usual behavioral indices of conditioned fear (Aggleton, 1992; Applegate, Frysinger, Kapp, \& Gallagher, 1982; Applegate, Kapp, Underwood, \& McNall, 1983; Canli \& Brown, 1996; Gallagher \& Chiba, 1996; Gallagher, Kapp, McNall, \& Pascoe, 1981; Kapp, Frysinger, Gallagher, \& Haselton, 1979; Kapp, Gallagher, Underwood, McNall, \& Whitehorn, 1982; Kapp, Supple, \& Whalen, 1994; Lam et al., 1996;
LeDoux, 1995; Maren, 1996; Scott et al., 1997; Whalen \& Kapp, 1991).

One limitation of this conventional model is that it fails to explain how associative LTP, which requires strict temporal contiguity (T. H. Brown, Chapman, Kairiss, \& Keenan, 1988; T. H. Brown, Ganong, Kairiss, Keenan, \& Kelso, 1989; Kelso et al., 1986), might be involved in encoding the long ISIs that occur during fear conditioning. In a more general context, this failure of associative LTP to account for temporal aspects of Pavlovian conditioning has already been noted as a serious problem (see Shors \& Matzel, 1997). We recently discovered that the cellular neurobiology and circuitry of the PR-ALa area furnish an exquisite potential solution to this dilemma (Faulkner, 1997; Faulkner, Tieu, \& Brown, 1997). In particular, we found PR cells that are capable of delaying firing for 3-4 sec in response to either a synaptic input or a current injection (Beggs, Moyer, \& Brown, 1997; Faulkner \& Brown, 1995, 1996, in press) and anatomical evidence suggesting that these cells are likely to be chained in series (Faulkner, 1997; Faulkner \& Brown, 1996, in press). On the basis of the neurobiology of the PR-ALa area, we previously outlined a conceptual model of how this region might participate in temporal encoding during Pavlovian fear conditioning (Faulkner, McGann, Tieu, \& Brown, 1997; Faulkner, Tieu, \& Brown, 1997; Tieu, Faulkner, \& Brown, 1996).

Here we elaborate on the conceptual model, develop a method for simulating the model in a way that captures the cellular and circuit dynamics, and show through simulations that the conceptual model does, in fact, work as desired, when cast in a quantitative form. There were four related goals. The first was to create and then evaluate the circuit-simulation software. The second was to show through simulations how the neurobiological circuitry could encode and accurately learn long ISIs (we looked at the range of $0.5-16 \mathrm{sec}$ ) using a standard Hebbian synaptic mechanism. The third goal was to determine whether this temporal learning mechanism naturally conforms to a Weber-like law, which quantifies the manner in which the learning accuracy should decrease as the training ISI increases. The fourth goal was to evaluate through simulations the more specific proposal that Weber-like learning accuracy emerges from intrinsic noise.

\section{EXPERIMENTAL AND CONCEPTUAL BACKGROUND}

The PR-ALa region in the rat appears to play an important role in certain aspects of learning and/or performance in fear conditioning (Corodimas \& LeDoux, 1995; Falls, Bakken, \& Heldt, 1997; LeDoux, 1995; Maren, 1996; Rosen et al., 1992). This laboratory has been investigating the physiological and anatomical properties of PR-ALa neurons (Beggs et al., 1997; Chapman et al., 1990; Faulkner \& Brown, 1995, 1996, in press) in order 
to understand how the circuitry might participate in Pavlovian fear conditioning (Canli \& Brown, 1996; Faulkner, McGann, et al., 1997; Faulkner, Tieu, \& Brown, 1997; Lam et al., 1996; Tieu et al., 1996). Of particular relevance to CS processing in fear conditioning, the PR is known to receive sensory inputs from both auditory (Deacon, Eichenbaum, Rosenberg, \& Eckmann, 1983; Inagaki, Matsuda, Nakai, \& Takagi, 1990; Mascagni, McDonald, \& Coleman, 1993; Romanski \& LeDoux, 1993) and visual cortices (Miller \& Vogt, 1984) and to project strongly to the ALa (McDonald \& Jackson, 1987; Romanski \& LeDoux, 1993; Turner \& Zimmer, 1984). The ALa, in turn, produces some of its effects via projections to the central nucleus of the amygdala (ACe; Krettek \& Price, 1978; Stefanacci et al., 1992; reviewed in Faulkner, 1997).

\section{Physiology and Anatomy of the PR-ALa Area}

The cellular anatomy and physiology of the PR-ALa area in the rat was recently studied in brain slices, using whole-cell recordings from visually preselected neurons (Faulkner, 1997; Faulkner \& Brown, 1995, 1996, in press). ${ }^{1}$ The patch pipettes contained biocytin, which enabled subsequent morphometric analysis of the recorded neuron. On the basis of characteristic responses to outward current steps, most of the cells in the PR could be classified into four main neurophysiological typesfast-spiking (FS), regular-spiking (RS), late-spiking (LS), and burst-spiking (BS). The cellular neurophysiology and neuroanatomy were incorporated into a conceptual model, schematized in Figure $1 \mathrm{~A}$, based on the dynamics of three of the four primary cell types and their interconnections (Faulkner \& Brown, 1996; Faulkner, Tieu, \& Brown, 1997; Tieu et al., 1996).

Using video microscopy, layer I of the PR could be seen to contain numerous afferents, dendrites of deeplayer cells, and small FS neurons, which we presume to be inhibitory. The small dendritic trees of the FS neurons were restricted to layer I, and the axons were collateralized extensively within the layer. FS neurons were seen in every layer of the PR (as well as in the ALa) and could maintain firing at frequencies over $100 \mathrm{~Hz}$ for a second or more (Figure 1B, FS) without exhibiting accommodation (i.e., the intervals between successive spikes did not change). Layer II/III was replete with RS and LS pyramidal neurons whose dendritic arbors branched extensively in layers I and II/III. The spiny dendrites of some of these layer II/III neurons can be presumed to receive CS-generated input from adjacent sensory cortical regions.

In response to a just-threshold current step, LS neurons typically delay firing for 1-3 sec (LS in Figure 1B; Figure $3 \mathrm{C}$ ), but much longer delays have been seen (Beggs et al., 1997; Faulkner, 1997; Faulkner \& Brown, 1995, 1996, in press). The firing onset latency depends on both the particular cell and the strength of the depolarizing current. With long current pulses, LS cells may exhibit anti-accommodation (the successive intervals between action potentials in a train become shorter; see Figure 3C). Axons of layer II/III LS cells collateralize extensively within the layer and also project to layers $V$ and VI. The within-layer projections are consistent with the possibility that $L S$ cells are connected to each other in chains or even loops (see Figure 1A). Because firing in a LS neuron can be delayed by several seconds, even short chains or small loops of LS cells could furnish large temporal delays. Layer II/III RS neurons do not exhibit this delayed spiking (Figure 1B, RS). In response to a long, depolarizing current step, RS cells show accommodation (successive intervals between action potentials become longer until the cell ultimately ceases firing; see Figure 1B, RS). The axons of these cells collateralize within the layer and also project to deeper layers of the PR.

There is no layer IV in PR. In layer V, RS and BS cells are prevalent, but we have not seen LS cells. Because the conceptual model (Figure 1A) does not include BS cells (because of uncertainty about their axonal projections), they are not further discussed here. The axons of RS neurons in layer V sometimes project back to layers II/III, but they can also project to layer VI, the external capsule, or the ALa. Among the large pyramidal cells, those of superficial layer $\mathrm{V}$ have apical dendritic tufts that spread to the outer edge of layer I. Their primary axons have been followed for over a millimeter, traveling in the external capsule with collaterals projecting throughout the PR. On the other hand, the dendrites of the deep layer $\mathrm{V}$ pyramids typically do not reach layer I, and their axons commonly project to the ALa.

LS neurons with ovoid somata are frequently found clustered in nests within layer VI. Although the axons of these LS cells generally collateralize in layer VI (suggestive of local chains or loops), these cells have also been seen sending their axons into the ALa, as well as to more superficial layers of the PR. The latter projections again are consistent with the idea of a reverberatory loop or a chain between the superficial and the deep layers. RS cells are also found in layer VI.

All of the firing properties seen in the PR were also observed in the ALa. Among the RS cells in the ALa, the extent of accommodation was sometimes greater than that seen in the PR. In extreme cases, the cells could be made to fire only once or twice to a current step. These extreme cases are called single-spiking (SS) neurons (Figure 1B, SS). This laboratory previously reported SS neurons in the ALa using microelectrodes (Chapman et al., 1990). In that same study, we also demonstrated, under current- and voltage-clamp conditions, that LTP can be induced in the ALa. Associative LTP (Barrionuevo \& Brown, 1983; Kelso \& Brown, 1986) in the ALa plays a key role in much of the contemporary thinking about fear conditioning (Chapman et al., 1990; Fanselow, 1998; Faulkner, Tieu, \& Brown, 1997; Lam et al., 1996; LeDoux, 1995; Maren, 1996). 


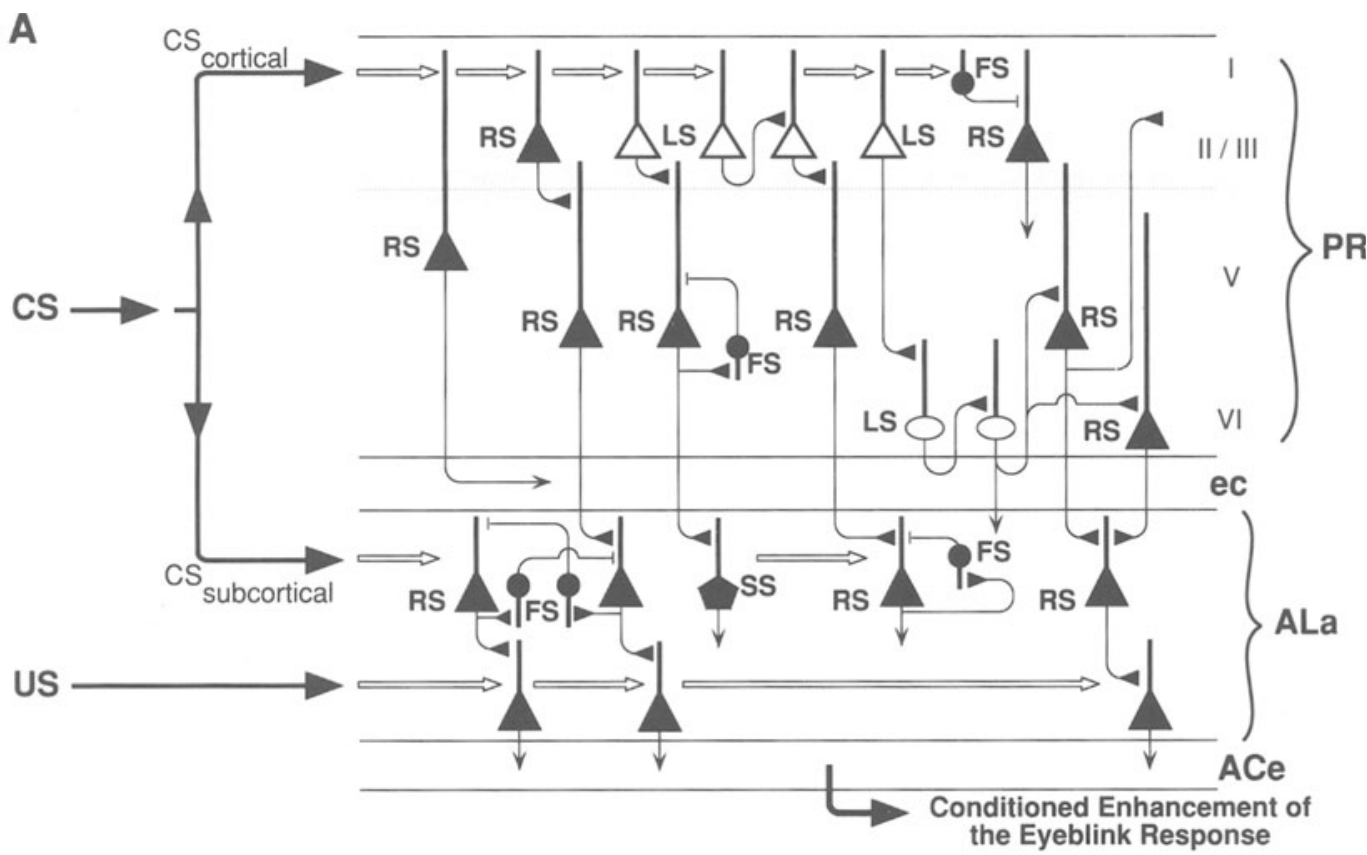

B
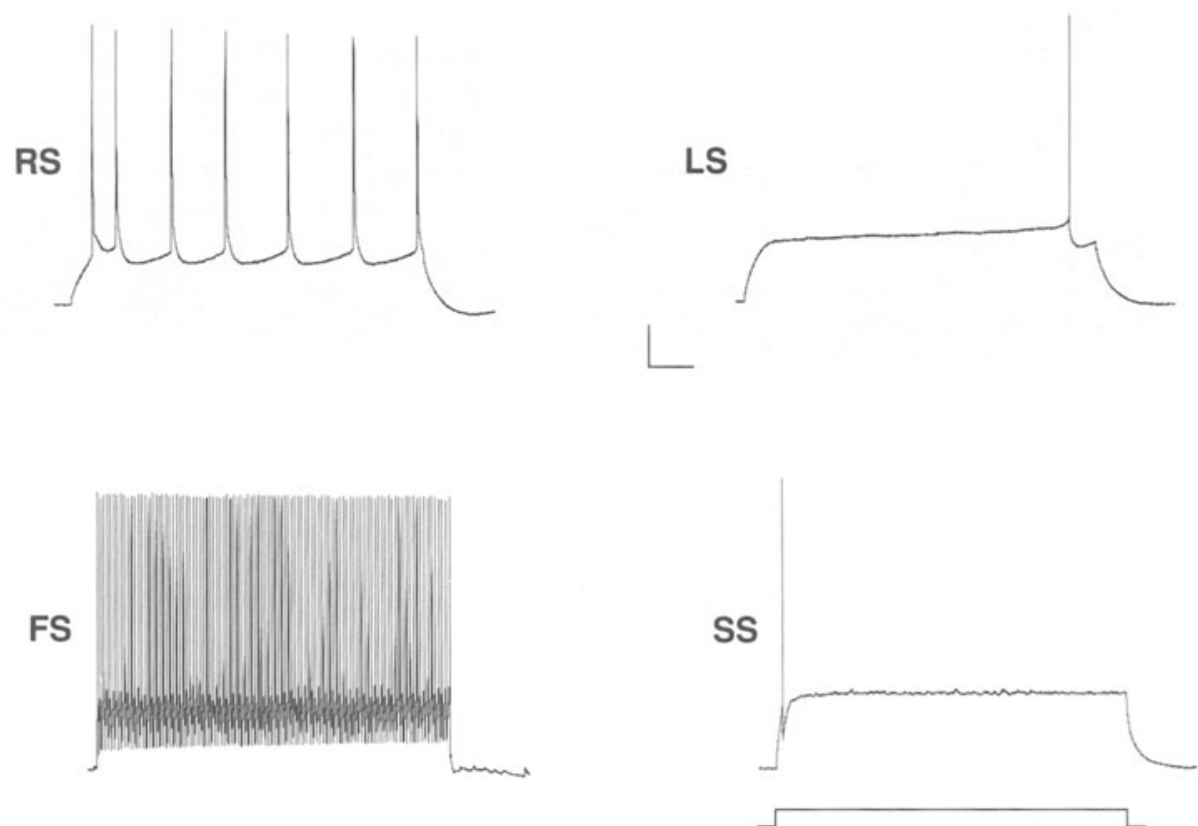

Figure 1. Neurons and circuitry proposed to participate in processing conditioned stimulus (CS) information en route to the lateral nucleus of the amygdala. (A) CS and unconditioned stimulus (US) inputs are indicated by filled arrows (before entering the perirhinal-amygdala system) and by hollow arrows (after entering the system). Excitatory synapses are indicated by solid triangular expansions and inhibitory synapses by short line segments perpendicular to the axon. Abbreviations: PR, perirhinal cortex; ec, external capsule; ALa, lateral nucleus of the amygdala; ACe, central nucleus of the amygdala; LS, late-spiking cell; RS, regular-spiking cell; FS, fast-spiking cell; SS, single spiking neuron. (B) Whole-cell recordings from PR and ALa neurons illustrating four basic firing patterns in the diagram of the conceptual model. The spiking types are indicated by their two-letter abbreviations, given above. Calibration scale: $20 \mathrm{mV}, 200 \mathrm{msec}$ (LS and FS); $20 \mathrm{mV}, 100 \mathrm{msec}$ (RS and SS). The depolarizing current step is shown only for the SS cell (lower trace). Modified from Faulkner (1997). 


\section{Conceptual Model}

The cellular anatomy and physiology of the PR-ALa region furnished the foundation for a conceptual model of temporal encoding and learning in fear conditioning (schematized in Figure 1A; Faulkner, 1997; Faulkner, McGann, et al., 1997; Faulkner, Tieu, \& Brown, 1997; Tieu et al., 1996). In this conceptual model, elaborated here, the firing properties and circuit-level organization of PR neurons provide a spectrum of delays, relative to the CS onset, in the activity transmitted to the ALa. Recall that activity induced by the CS and US is presumed to converge in the ALa, resulting in associative LTP (Chapman et al., 1990; Kelso \& Brown, 1986; Lam et al., 1996; Maren, 1996; Quirk et al., 1995; Romanski et al., 1993). The range of delays in firing onsets observed in the ALa results from various combinations of cell types and their interconnections in the $P R$. The firing in the $\mathrm{ALa}$, once initiated, is presumed to terminate because of a combination of spike-frequency accommodation (in RS cells) and synaptic inhibition (from FS cells), resulting in temporal windows of activity.

Learning is proposed to result from a Hebbian synaptic modification in the ALa whenever there is conjoint presynaptic and postsynaptic activity. It is known that Hebbian synapses exist (T. H. Brown et al., 1990; Kelso et al., 1986; Kirkwood \& Bear, 1994; Magee \& Johnston, 1997) and can give rise to associative LTP (Barrionuevo \& Brown, 1983; T. H. Brown et al., 1990; Kelso \& Brown, 1986; Levy \& Steward, 1979). It is also known that LTP occurs in the ALa (Chapman et al., 1990; Rogan \& LeDoux, 1995). By analogy to associative LTP (Kelso \& Brown, 1986; Kelso et al., 1986), the CS is presumed to generate the requisite presynaptic activity, and the US is presumed to generate the required postsynaptic activity. Because the exact form of the synaptic modification rule in the ALa is not yet known, here we assume a BCM-type learning rule (Figure 4; "BCM" refers to the authors who first suggested this rule: Bienenstock, Cooper, \& Monro, 1982) because it is biologically plausible and allows for bidirectional synaptic change (see also Bear \& Abraham, 1996; Bear \& Malenka, 1994; T.H. Brown \& Faulkner, 1998; T. H. Brown et al., 1990; Heynen, Abraham, \& Bear, 1996; Hua \& Houk, 1997; Magee \& Johnston, 1997). When a CS is explicitly paired with a US, LTP occurs in the ALa in only those cells that were active at the time of the US.

The increased activity in the ALa is presumed to increase the output from the $\mathrm{ACe}$, which is believed to be responsible for generating certain CRs (Canli \& Brown, 1996; Kapp et al., 1979; Kapp et al., 1982; Kapp et al., 1994; Kapp et al., 1992; Lam et al., 1996; LeDoux, 1993, 1995). In accordance with the BCM rule (Figure 4), longterm synaptic depression (LTD) can, in principle, occur (Debanne, Gähwiler, \& Thompson, 1994; Kirkwood, Lee, \& Bear, 1995; Mulkey, Herron, \& Malenka, 1993; Oliet, Malenka, \& Nicoll, 1997) during nonreinforced (extinction) trials when the CS input is active but the level of post- synaptic activity is low (because there is no strong input from the US).

The conceptual model assumes that the CS is most realistically represented by the spiking pattern among a large set of input neurons. More specifically, CS-related neuronal firing is viewed as a time-varying input activity vector (Mauk \& Donegan, 1997). The FS cells in layer I mediate feedforward inhibition, tending to "normalize" these time-varying input vectors. FS cells also mediate feedback and lateral inhibition.

\section{SIMULATION METHODS}

In this investigation, we simulated a simplified version of the conceptual model (Faulkner, Tieu, \& Brown, 1997), subjected it to either of two training regimes, and then tested it to see what it had learned. The program, YNET, was written in $\mathrm{C}$ and run on a Sun Ultra 1 workstation. The circuit that we used for simulations (see Figure 5) is simpler than the conceptual model in three key respects. Our computational model (1) is purely feedforward, (2) consists of only RS and LS cell types, and (3) represents the CS by activity on a single input line. The exclusion of FS cells from the model does not pose a problem, because (1) the simulated circuit does not include recurrent excitation and (2) the accommodation of our model RS cells is sufficient to terminate firing.

\section{Neurons}

The behavior of our model neurons closely matches the dynamics we see in our in vitro neurophysiological recordings, in terms of the relationship between their inputs and their (1) output frequency, (2) delay in firing the first spike, and (3) spike-frequency accommodation properties. Although we do not use conductances in these model neurons, the symbols and formalisms are similar where appropriate (see Appendices A-C, E). RS and LS neurons can be thought of as consisting of five modules (accumulator, accommodator, frequency generator, spike generator, and state), connected as illustrated in Figure 2.

The state is a collection of variables that holds information about the cell, such as the time of the cell's previous spike and the values produced by the other modules. Input to the cell is handled by the accumulator, which is implemented as a recurrence relation that rises and falls on the basis of its previous value, the strength of the input, a charging time constant, and an accommodation term (determined by the accommodator). The accommodator works differently for each cell type, causing RS cells to accommodate and LS cells to anti-accommodate (FS cells lack an accommodator and are nonaccommodating). The frequency generator produces the cell's spike-frequency value on the basis of the level of the accumulator, and the spike generator uses this spike-frequency value to determine when the cell spikes. The precise equations defining the modules and their interactions are given in Appendices A-C (see also Table El of Appendix E). 


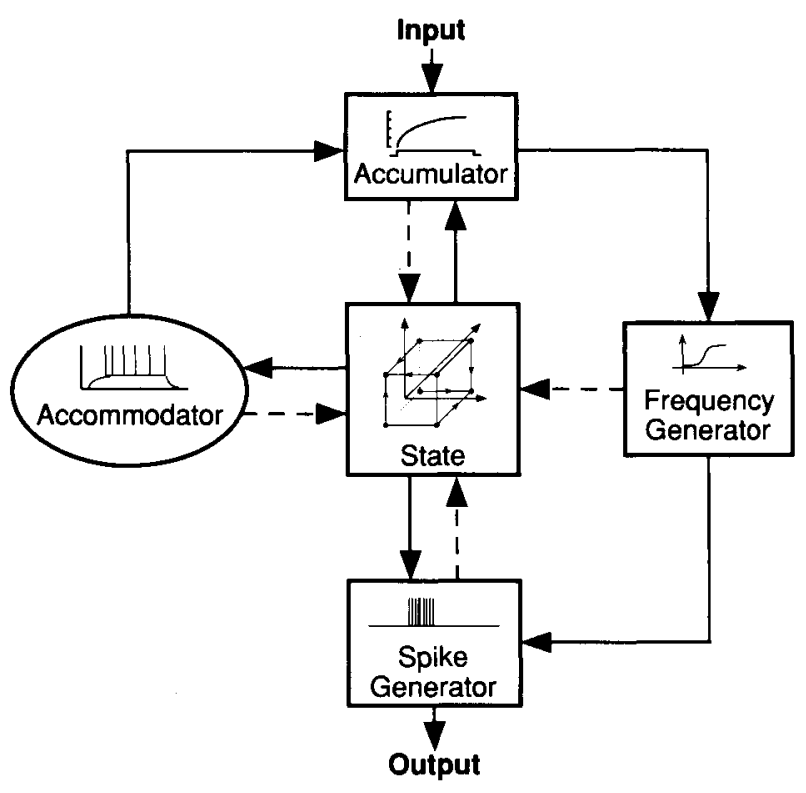

Figure 2. Information flow and control among the modules that generate the dynamics of each type of model neuron (described in the text). The accumulator, accommodator, frequency generator, and spike generator are algorithmic blocks of code. The state is a data structure that is updated at every time step. The accumulator, accommodator, and spike generator read from and write to the state. The frequency generator only writes to the state. A pair of solid and dashed lines indicates that the outer module receives input before writing to the state. Fast-spiking neurons lack an accommodator module. Regular-spiking and late-spiking neurons have very different accommodators.

It is important to emphasize the dynamic nature of our model neurons. The values produced by the modules and held in the state are constantly changing because of varying input and changes occurring within the neuron itself. In the following descriptions of each cell type, it is assumed for simplicity that the cell is in a noise-free environment and that the stream of input to the cell, once initiated, is continuous and unvarying. Later, we will return to the issue of noise.

Fast-spiking neurons. FS neurons are the simplest of the three cell types and are the most similar to a conventional integrate-and-fire unit (see Figure 3A). We created only one type of FS cell, which is described and illustrated here, even though it is not used in the present circuitlevel simulations. These FS neurons and the formalisms that define them furnish a simple reference point for comparison. It is necessary to include them in recurrent networks to maintain circuit stability. The precise equations and parameter values governing FS cells are given in Appendices A and E (Table E1).

Unlike the RS and LS neurons, the representation of FS neurons does not include an accommodator module (Figure 2), so these model neurons do not exhibit accommodation (Figure 3A). Prior to receiving input, the accumulator of a FS neuron is empty (its value is zero), the frequency and spike generators are inactive (their values are zero), and the values held in the state remain unaltered.
When the cell begins receiving input, the accumulator rapidly increases until it reaches the neuron's spiking threshold (given typical inputs, the rise to threshold takes only $\sim 10 \mathrm{msec}$ for FS cells). Once spiking threshold is reached, all of the modules are affected: The value of the accumulator is boosted so that it equals the magnitude of the input; the frequency generator is activated and determines the neuron's spiking frequency; the spike generator changes its value from 0 to 1 , representing the physiological process of the neuron spiking; and the values in the state are updated accordingly.

As long as the stream of input remains unbroken, the accumulator is set to the value of the input on each time step. In simulations, the input can drop below spiking threshold for long periods of time or even disappear completely. In such cases, the value of the accumulator is set to a subthreshold value, and the frequency and spike generators are set to zero until the accumulator once again rises above the spiking threshold. In the simpler scenario, where the accumulator remains above spiking threshold, the frequency generator stays active and generates the neuron's instantaneous spiking frequency value on each time step. The values calculated by the frequency generator are completely dependent on the value of the accumulator, which itself mirrors the magnitude of the input. The frequency generator is a sigmoid function, so that once a maximum frequency is reached, increasing the intensity of the input cannot further increase the neuron's spiking frequency (see Figure 6). Following the initial spike, the spike generator emits additional spikes on certain time steps in accordance with the frequency values produced by the frequency generator. The output of the spike generator is the only external output produced by the model neuron.

Regular-spiking neurons. The functioning of RS neurons is similar to that of FS neurons, with one important addition: The representation of RS cells includes a fifth module, called the accommodator (Figure 2), which is responsible for the accommodation properties exhibited by RS cells (Figure 3B). Appendix B gives a formal account of the implementation of RS cells (see also Table E1 of Appendix E). For RS neurons, the value of the accumulator is determined by the state and by a recurrence relation that depends on the input, the value of the accumulator on the previous time step, and the value of the accommodator. The accommodator is set to zero at the beginning of a trial, and its value remains close to zero until the accumulator reaches the cell's spike threshold. The accumulator takes significantly longer to reach spike threshold for RS cells than for FS cells.

Once the neuron has begun spiking, the accommodator grows at an accelerated pace, causing the accumulator to decline slowly. Decreases in the accumulator cause the frequency generator to produce lower values for the neuron's spiking frequency, resulting in lower frequency output from the spike generator. Eventually, the accommodator causes the accumulator to fall below threshold, which immediately forces the frequency generator to zero, causing spiking to terminate (see Figure 3B). Deactiva- 

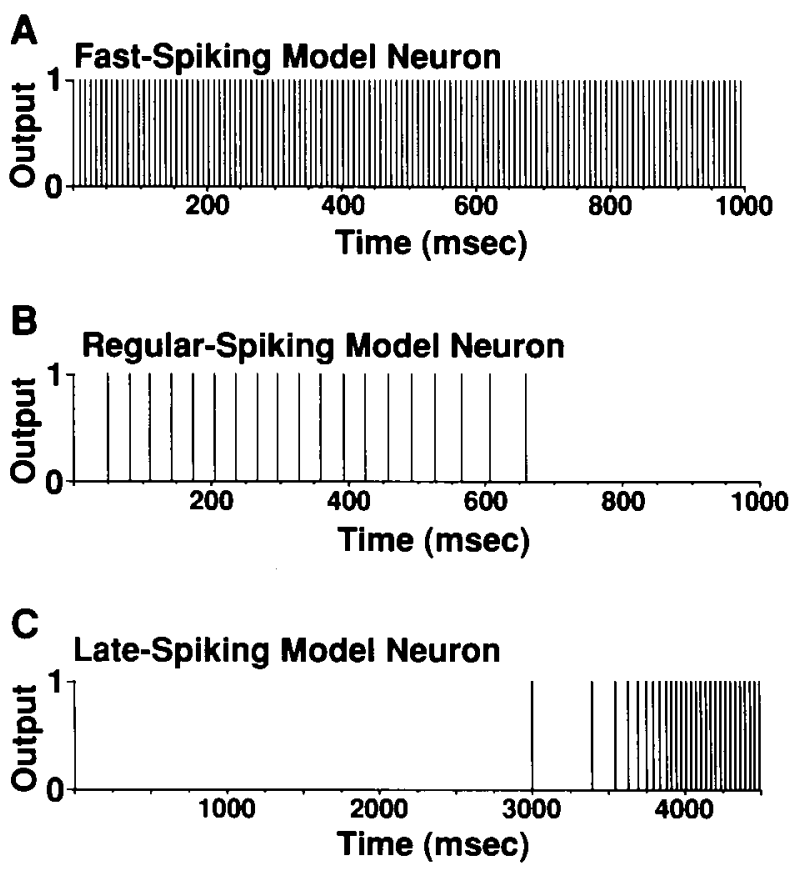

Figure 3. Spiking patterns for the three types of model neurons. (A) Fast-spiking cells exhibit the shortest latency to fire and are nonaccommodating. (B) Regular-spiking cells receiving direct conditioned stimulus input delay their spiking for $50 \mathrm{msec}$ and then accommodate (see also Figure 5 and text). (C) Latespiking 4 cells show the longest delay among all the model neurons used in the simulations, and their spike trains exhibit antiaccommodation.

tion of the frequency generator prevents the spike generator from producing any further output, so that the neuron ceases spiking for the remainder of the trial. As a result of these interactions, in response to a continuous and fixed suprathreshold input, the intervals between successive spikes grow longer until the neuron eventually stops firing.

In our physiological recordings, there are variations among RS cells in terms of the charging time constant (which determines the amount of time before the accumulator reaches spike threshold). In the equations for RS cells (Appendix B), the charging time constant $\left(\tau_{A}\right)$ appears in the denominator of the recurrence relation for the accumulator. To make our model RS neurons more closely emulate our recorded RS neurons, we varied $\tau_{\mathrm{A}}$ to create four subtypes of RS cells (denoted RS1-RS4) that differed in their delays before firing the first spike. The value of $\tau_{\mathrm{A}}$ is the only source of variation among the RS cells used in these simulations. Other things being equal, larger values of $\tau_{\mathrm{A}}$ cause longer charging times before the neuron begins spiking. Among the different subtypes of RS cells, the charging time necessary to reach spike threshold ranges from 50 to $300 \mathrm{msec}$ in response to CS input. Table E1 in Appendix E lists the values of the parameters used to create the four types of RS cells. For comparison purposes, Table E1 also includes the parameter values for FS and LS neurons (parameters not used are denoted NA).
Late-spiking neurons. LS cells are represented by equations similar to those used for RS cells, but the accommodator is implemented differently, causing LS cells to exhibit anti-accommodation (successive intervals between spikes become progressively shorter; see Figure 3C). Recall that, in RS neurons, the accommodator is initially zero and begins to grow when the neuron starts firing; with continuous firing, the accommodator's rate of growth accelerates until the accommodator terminates the spike train. In LS cells, the accommodator acts in an opposite manner. When an LS cell begins receiving input (assume for simplicity that it is continuous and unvarying), the accommodator rapidly rises to a large value (before the accumulator reaches spiking threshold), so that the rate of growth of the accumulator is dramatically reduced. Over a time span ranging from hundreds to thousands of milliseconds, the accumulator gradually rises while the accommodator slowly ebbs, until the accumulator finally reaches the neuron's spiking threshold. This activates the frequency and spike generators, and the neuron fires for the first time. Notice the long delay to the first spike in the model LS neurons (Figure 3C), in comparison with the delays seen in the model FS and RS neurons (Figures $3 \mathrm{~A}$ and $3 \mathrm{~B}$, respectively). These firing patterns resemble those observed in our whole-cell recordings (Faulkner \& Brown, 1995, 1996, in press).

Following the initial spike, the accommodator's value falls quickly and asymptotically approaches zero. The decline of the accommodator allows the accumulator to increase until it approaches the value of the input. As the accumulator increases, so do the spike-frequency values produced by the frequency generator, causing the spike generator to fire the cell at an increasingly fast pace. The process described above illustrates the two fundamental features that distinguish LS cells from the other cell types (see Figure 3): (1) As the name implies, LS cells undergo a much longer delay before firing their first spikes, and (2) LS cells demonstrate anti-accommodation. The first difference is caused by the steep initial increase in the accommodator in response to input. The second difference is a consequence of the decrease in the accommodator following the first spike.

In our physiological recordings, LS neurons demonstrated significantly longer output latencies (relative to those of RS neurons) for any given input (Faulkner, 1997; Faulkner \& Brown, in press). These long spike delays are possibly due to a slowly inactivating potassium current ( $I_{\mathrm{KD}}$, which is governed in the model neurons by $\tau_{h \text {, max }}$ in Appendix C; see Johnston \& Wu, 1995). We therefore varied the parameter controlling the decay of the accommodator $\left(\tau_{h, \max }\right)$ to create four subtypes of LS cells that differed in their delays before the initial spike. Table El in Appendix $\mathrm{E}$ lists the values of the parameters used to create the four types of LS cells, denoted LS1-LS4.

\section{Synapses}

Every synapse was given a weight that determined the magnitude of the input received by the postsynaptic neuron when the corresponding presynaptic neuron spiked. 


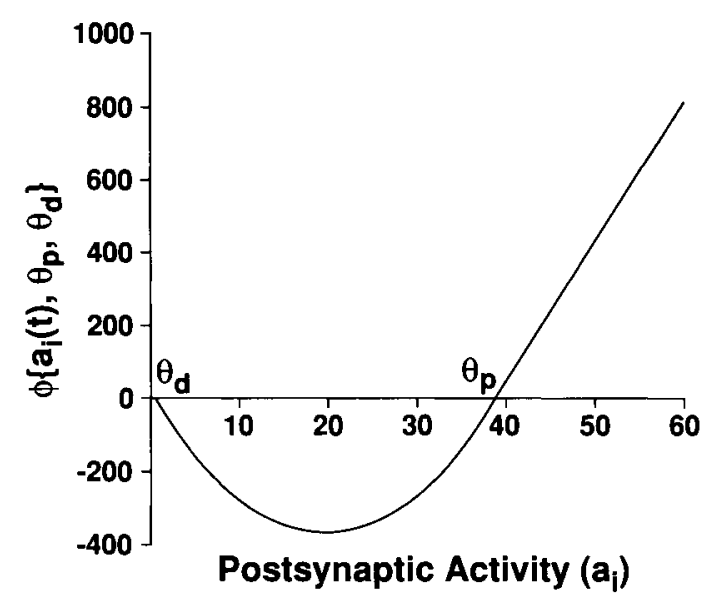

Figure 4. BCM-type $\phi-a_{i}$ relationship. The postsynaptic activity level $a_{i}$ determines the value of the $\phi$ function. The $\phi$ function, in conjunction with the presynaptic activity level $a_{j}$, determines the synaptic modification (see Appendix D). Low levels of postsynaptic activity $\left(a_{i}(t) \leq \theta_{d}\right)$ result in no change in synaptic strength $(\phi=0)$, regardless of the level of presynaptic activity. Somewhat higher postsynaptic activity levels $\left(\theta_{d}<a_{i}(t)<\theta_{p}\right)$ result in synaptic depression $(\phi<0)$ if there is presynaptic activity $\left(a_{j}>0\right)$. Still greater postsynaptic activity $\left(a_{i}(t)>\theta_{p}\right)$ causes synaptic potentiation $(\phi>0)$ if there is presynaptic activity. For any given level of postsynaptic activity $a_{i}$, the synaptic change depends on the level of presynaptic activity $a_{j}$.

Synaptic input to each neuron equaled the summed products of the presynaptic activities and their corresponding weights. The equations and constants used to implement synapses are given in Appendix D and Table E2 of Appendix E.

Noise. In addition to the weighted sum described above, in each synapse we introduced a Gaussian random variable, $z$, to represent noise. There are several natural noise sources. Intrinsic synaptic noise is due to probabilistic fluctuations in the number of quantal packets of transmitter released and to random variations in the response to each released quantum (Stevens, 1996; Stevens \& Wang, 1994; Xiang, Greenwood, Kairiss, \& Brown, 1994), as well as to fluctuations in the number of active synapses at any given time. The synaptic noise we introduced represented a combination of these three local synaptic noise sources. Other noise components could arise from fluctuations in CS processing associated with such systems-level phenomena as changes in attention, response-produced stimulus changes, or variations in relative salience. Although these systems-level sources could be included in the CS input to the circuit, in the simulations that follow we assumed that the CS was presented to the PR-ALa circuitry in a noise-free fashion, so that the local noise contributions could be more easily evaluated.

In the first set of simulations that included noise, a new value of $z$ was selected at each 1 -msec time step, corresponding to a high-frequency noise source. In the second such set of simulations, lower frequency noise was injected by changing the value of $z$ on randomly selected time steps determined from a uniform distribution with limits of $50-$ and $250-\mathrm{msec}$ time steps. These results were compared and analyzed in relationship to noise-free simulations. What we will see is that only noise in the appropriate frequency range resulted in Weber-like temporal learning of the CS-US relationship.

Synaptic modifiability. The weights of some model synapses were unmodifiable (nonplastic), whereas others exhibited bidirectional "Hebbian" (plastic) changes (T. H. Brown et al., 1990). The Hebbian synaptic modification algorithm we used was a variant of the $\mathrm{BCM}$ learning rule (Bienenstock et al., 1982; see Figure 4) that allows synapses to potentiate (LTP) or depress (LTD), depending on pre- and postsynaptic activity levels (Bear \& Abraham, 1996; Bear \& Malenka, 1994; T. H. Brown et al., 1990; Heynen et al., 1996; Kirkwood et al., 1995; Magee \& Johnston, 1997; Neveu \& Zucker, 1996; Oliet, Malenka, \& Nicoll, 1996). There appears to be more than one form of LTP/LTD (T. H. Brown et al., 1989; Cavus \& Teyler, 1996; Grover \& Teyler, 1990; Nicoll \& Malenka, 1995; Oliet et al., 1997; Teyler et al., 1994), and the exact synaptic modification rules or algorithms (T. H. Brown et al., 1990) have yet to be elucidated.

Potentiation and depression. In our version of the BCM-type learning rule, there are postsynaptic thresholds for synaptic potentiation $\left(\theta_{p}\right)$ and depression $\left(\theta_{d}\right.$; Bear \& Malenka, 1994; Bienenstock et al., 1982; Heynen et al., 1996; Neveu \& Zucker, 1996). The relationship between postsynaptic frequency and the resulting change in synaptic weight has the familiar BCM-type shape (Figure 4). For postsynaptic activity (firing frequency) values between $\theta_{d}$ and $\theta_{p}$, there is a parabolic region of depression, and for postsynaptic frequency values greater than $\theta_{p}$, there is a linear region of potentiation.

In the computational model, we introduced two additional variations to the basic BCM-type learning rule. The first is that different kinetics exist for synaptic potentiation and depression (see Appendix D and Table E2 of Appendix E). The second variation, made in agreement with physiological findings, is that limits (Appendices D and E) are imposed on the amount of change that can be produced by synaptic potentiation and depression (Bear \& Abraham, 1996; Bear \& Malenka, 1994; Heynen et al., 1996; Oliet et al., 1996). These limits prevent certain implausible and/or undesirable outcomes (T. H. Brown et al., 1990), such as weights that become extremely large or that change sign from positive to negative.

Threshold for potentiation. At plastic synapses, the value of the potentiation threshold, $\theta_{p}$, was set so that the US-produced postsynaptic activity would always be greater than $\theta_{p}$ and the maximum value of postsynaptic activity produced by the CS alone, after asymptotic learning, would be restricted to the following limits: $\theta_{A}<$ $a_{i}(t)<\theta_{p}$, where $\theta_{A}$ is the neuron's spiking threshold and $a_{i}(t)$ is the level of postsynaptic activity. The effect is that active plastic synapses associated with a nonreinforced CS-produced input will depress; those associated with a 


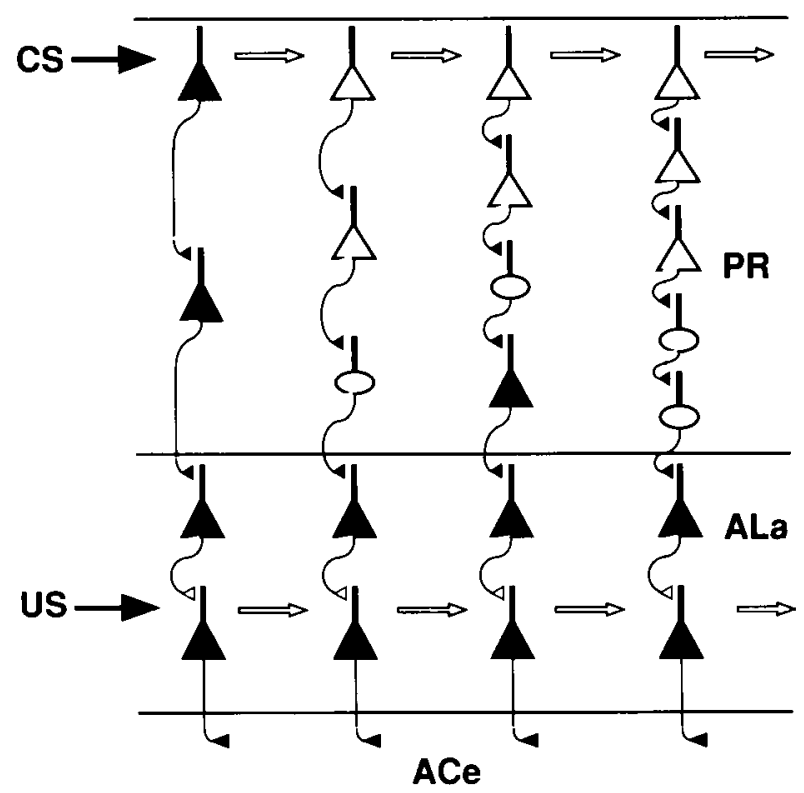

Figure 5. Network architecture. The network architecture was derived from a simplification of the neuronal relationships depicted in Figure $1 \mathrm{~A}$. Information about the conditioned stimulus (CS) is made available to the first neuron in each chain. The only synapses capable of Hebbian modifications are those between the first and the second rows of neurons in the lateral amygdala (ALa). The perirhinal cortex (PR)-ALa circuit is comprised of 189 chains of neurons, ranging in length from 4 to 16 neurons. Chains of PR neurons consisted of either regular-spiking (RS) cells (subtypes 1-4), late-spiking (LS) cells (subtypes 1-4), or a combination of both. The two layers of neurons in the ALa were composed of only RS1 cells. The solid triangles represent RS neumons, and the open patterns represent two of the various morphological types of LS neurons. ACe, central nucleus of the amygdala.

reinforced synaptic input will potentiate; ones that have potentiated sufficiently will be above the spiking threshold; and conditioning cannot cause the synapses to become self-potentiating in the absence of the US.

\section{Network Architecture}

The PR and the ALa include massive recurrent excitation, and recurrent circuits have some very desirable properties (see Anderson, 1985; Churchland, 1995; Hinton \& Anderson, 1981; Hopfield, 1984; Kohonen, 1984). However, in the interest of simplicity, the PR-ALa circuit used in this scaled-down computational model included solely feedforward connections and consisted of $\sim 1,600$ neurons. The architecture was arranged to emulate only the feedforward aspects of the biological circuitry by which CS information can flow through the $P R$ and activate neurons in the ALa (see Figure 5). Note that the ALa also received a direct US input. The output of the ALa was monitored and used as the dependent variable.

Perirhinal cortex. Altogether, $189 \mathrm{PR}$ model neurons received direct input from the $C S$, and each served as a starting-point for a chain of neurons. This resulted in 189 independent nonbranching chains of various lengths and cell types, as is suggested by the anatomy and physiology. Short-latency inputs from the PR to the ALa were furnished by short chains consisting largely of RS cells (see Figure 5). Recall that, for all types of neurons, output firing stops as soon as input ceases. Thus, it was impossible to build long chains of RS cells, because once any RS cell stopped firing because of accommodation (which happened in less than $1.3 \mathrm{sec}$, given a typical input), the chain was broken and all subsequent cells in the chain stopped firing.

Longer latency inputs to the ALa were formed by chains of LS cells associated with layers II/III and VI. Some chains of PR neurons included both RS and LS cells. Chains of PR neurons varied in length from a minimum of 2 neurons to a maximum of 14 neurons. Figure 5 illustrates four viable model neuron chains. Notice that the initial neuron in each chain is positioned to receive continuous CS input. The solid triangles represent RS cells, and the open patterns represent two of the morphological types of LS cells. Given a continuous input, chains of LS cells were capable of sustained firing (because LS cells anti-accommodate). The terminal neurons in all PR chains projected to the first layer of RS neurons in the ALa. In this way, synaptic activity traveled through the PR via neuronal chains of various lengths (numbers of neurons) and cell types, thus presenting CS input to RS neurons in the ALa at a spectrum of delays.

Lateral nucleus of the amygdala. The entire ALa was compressed into two layers of RSl cells, each containing 189 neurons. The PR input to the first layer of the ALa consisted of nonplastic synapses. The synaptic weight of all nonplastic synapses carrying CS information to the ALa was set to 30. The first layer of the ALa projected to the second layer of RS neurons via plastic (Hebbian) synapses. All the plastic synapses had a weight of 1 at the beginning of a conditioning session and were subject to minimum and maximum possible weights of 0 and 28 , respectively. There was no convergence or divergence between layers.

The RS neurons of the second layer of the ALa received direct US input in addition to activity from the first layer of the ALa. The synaptic weight of the US input was 60 , double that of the nonplastic synapses carrying CS information. The weight of the US input was, by design, sufficient to drive postsynaptic activity in the second layer RS neurons into the range of the $\mathrm{BCM}$ curve that allows induction of long-term synaptic potentiation (Figure 4; postsynaptic activity $>\theta_{p}$ ). Because of their accommodation properties, RS cells in the ALa served to limit the duration of activity from any given chain of PR neurons to a relatively brief window, despite constant input.

Associative LTP was restricted to the Hebbian synapses onto RS neurons in the second layer of the ALa; these RS neurons received simultaneous inputs representing both the CS and the US. Hebbian inputs are depicted in Figure 5 by unfilled (open) symbols for synapses, whereas nonplastic inputs are represented by filled (solid) symbols. The model did not include a representation of the 
subcortical CS input to the ALa (see Figure 1A), which is also thought to be involved in aspects of conditioned responding (LeDoux, 1995; Romanski \& LeDoux, 1992).

Limitations of a developing database. Although the scaled-down circuit used in this computational model places the essential temporal machinery in the PR and the essential plasticity in the ALa, this is simply a convenience and a reflection of our limited database of the cellular neurobiology of these brain regions. Both timing and plasticity may be operative in both regions, as well as other circuitry that may be relevant to fear conditioning. Indeed, it would be surprising if the ALa neurons were the only relevant site of convergence of CS and US information (see Cahill \& McGaugh, 1998; Killcross, Robbins, \& Everitt, 1997), although that is the implicit assumption of some contemporary conceptual models. It is important to recall that we already have evidence that the ALa contains all of the same cell types that exist in the PR, although the internal organization of the ALa is somewhat more difficult to grasp, because it does not have the convenient layering of the PR. Let us therefore reemphasize that we do not presume that learning and timing are spatially segregated, which was done here partly for convenience and partly because of limitations on biological data.

Multisynaptic model neurons. The simplicity and relatively small size of the circuitry created a problem that is not commonly encountered in mammalian neurobiology - namely, the need for one neuron to be able to fire another neuron. One presumes that synaptically-driven neuronal firing is normally dependent on convergent input from tens to hundreds of other presynaptic neurons. Without using very large circuits, an obvious solution to this problem is to make each synaptic weight unusually strong - to use "detonator synapses."

The alternative approach that we adopted seemed to work better. We simply increased the frequency of synaptic inputs to a postsynaptic cell to 150 times the value signaled by the frequency generator of the presynaptic neuron. The effect was that a single neuron could fire a postsynaptic cell by itself without "jolting" the circuit with periodic "detonator"-type synaptic inputs. This allowed our scaled-down model to make use of simple feedforward chains without the overhead associated with convergent input in a much larger circuit.

\section{Experimental Design}

Learning and testing. During the learning phase of the experiment, the circuit was exposed to either of two types of stimulus presentation. In the equivalent of an "experimental" or "conditioning" group, the CS and US were explicitly paired in a delay paradigm (six trials in one conditioning session). The US was always $0.5 \mathrm{sec}$ in duration; the interval between the CS onset and the US onset, or the ISI, was varied among different conditioning experiments but was kept constant for all six trials within a given conditioning session; and the CS and US coterminated. Thirteen different conditioning sessions were conducted, with the ISIs ranging from 0.5 to $16 \mathrm{sec}$ across different sessions.

In the equivalent of a "control" or "pseudoconditioning" group, the CS and US durations were the same as those in the experimental group and were presented the same number of times; however, they were explicitly unpaired (they did not overlap temporally). Specifically, the US onset occurred $1 \mathrm{sec}$ after the CS offset. In both groups, each trial continued for $2 \mathrm{sec}$ after the US offset, which was more than enough time for the circuit to settle to an inactive state.

During the testing phase of the experiment, the CS was presented alone (without the US) continuously for $30 \mathrm{sec}$. Each testing trial ended $2 \mathrm{sec}$ after the CS offset. The time of occurrence of all unit activity in the output from the ALa was noted during the testing trial (see below).

Dependent variable. Learning was monitored by the output from the second layer of the ALa neurons. The time of occurrence of all spikes in these ALa neurons was recorded. Associative learning was assessed in terms of differences between the experimental and the control circumstances in terms of CS-produced unit firing in these ALa output neurons. Temporal encoding was evaluated in terms of the relationship between the ISIs used during associative conditioning and the resulting timing of CSproduced output from the the ALa neurons. This relationship is described via a scatterplot, its regression equation, and the associated square of the Pearson productmoment correlation coefficient $\left(r^{2}\right.$, the coefficient of determination).

To determine whether temporal learning followed a Weber-like law, we examined response accuracy as a function of the ISI used during conditioning. The possibility of Weber-like temporal learning accuracy was examined in scatterplots and by quantifying the relationship between the coefficient of variation of the firing latency and the mean firing latency for all spikes in all output neurons for each of the 13 ISIs used during conditioning (Gibbon, 1977; Gibbon et al., 1997).

\section{SIMULATION RESULTS}

We will start by describing the dynamical properties of the neurons and exploring the temporal aspects of the circuit-level performance that enable temporal encoding. This will be followed by a demonstration that the model does indeed accurately encode time over a long range of ISIs. Finally, we will emphasize the importance of noise, interacting with intrinsic circuit dynamics, in causing Weber-like temporal learning (see Gibbon, 1977; Gibbon et al., 1997).

\section{Neuronal Dynamics}

We created three basic types of model neurons-FS, RS, and LS (see firing patterns in Figure 3)--but in the circuit simulations described below, only the RS and LS cells were used (see Figure 5). Because all three cell types 

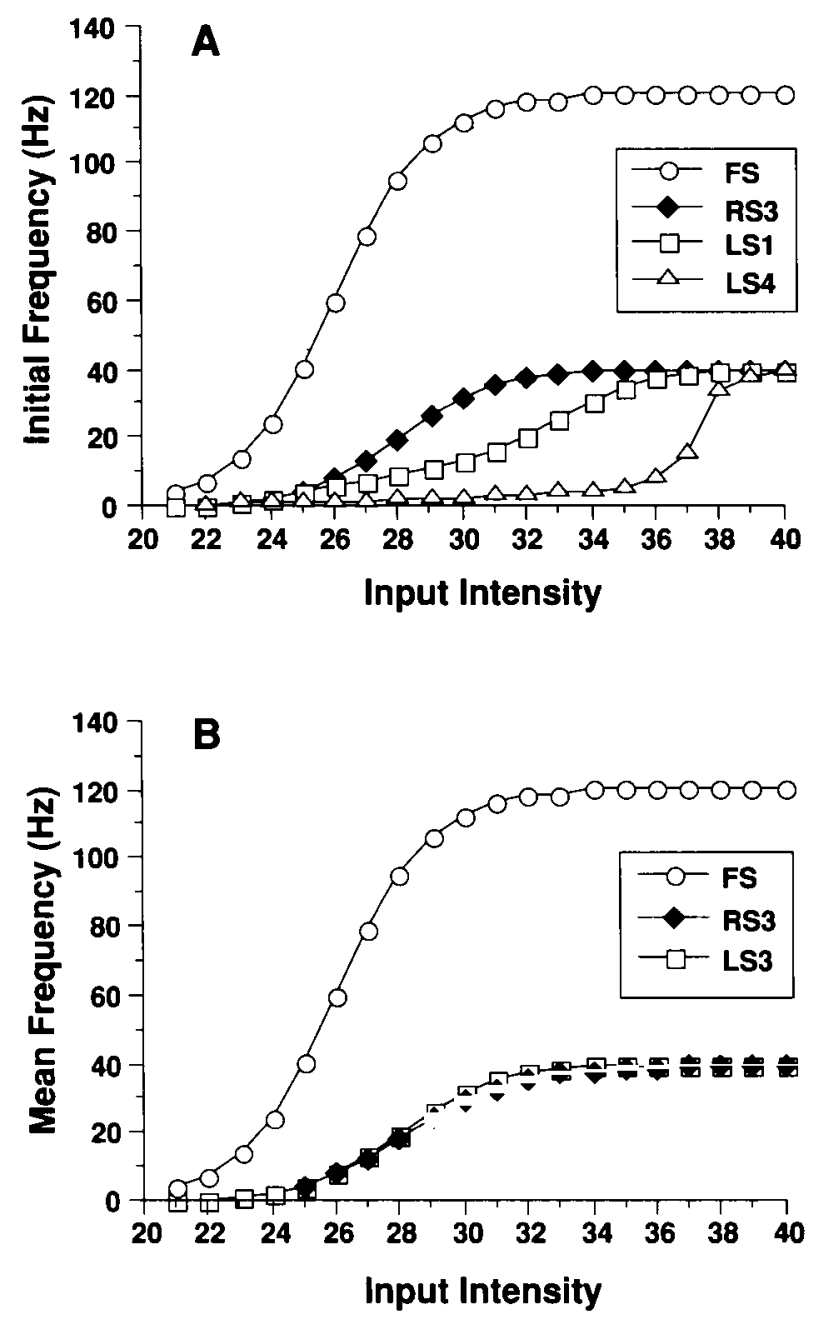

Figure 6. Spike firing frequency as a function of input intensity. The relationship between firing frequency $(F)$ and input intensity (I) has a sigmoidal shape for all three types of neurons (see the text for elaboration). Two kinds of $F-I$ plots are shown. It is worth noting that an input of 25 or larger was required to generate more than one spike in regular-spiking 3 (RS3) cells (hence, no RS3 data points for $I<25$ ). (A) In the first type of $F-I$ plot, the ordinate is initial firing frequency (reciprocal of the interval between the first two spikes). (B) In the second type of $F-I$ plot, the ordinate is the mean firing frequency (time average during a spike train).

will be necessary in subsequent studies and will presumably be useful to others interested in network-level simulations using model neurons with realistic inputoutput dynamics, all three types are compared here. The behavior of our model neurons constitutes a reasonable approximation of what we observed in our physiological experiments on brain slices (Faulkner, 1997; Faulkner \& Brown, 1995, 1996, in press). Here, the dynamics of the model neurons are captured in the same kinds of plots used to characterize the input-output relationships of recorded PR-ALa neurons.
It is clear from neurophysiological studies that there is considerable variability within both the RS and the LS categories, and we found that small parametric variations allowed us to capture some of this variability in the form of four subtypes of RS neurons (RS1-RS4) and four subtypes of LS neurons (LS1-LS4), as was described earlier. Because the FS cells are most similar to conventional integrate-and-fire units, they serve as a convenient point of comparison with the RS and LS cells.

Frequency versus input intensity relationships. One of the most characteristic features of a neuron is the relationship between its input magnitude $(I)$ and its output firing frequency $(F)$. Input intensity for the model neurons is a dimensionless variable (which ranges in these experiments between 0 and $\sim 98$ ) that represents the sum of the inputs to a neuron (including noise; specified in Equations 23 and 24 of Appendix D). ${ }^{2}$ In neurophysiological studies, the firing frequency is taken either as the mean over some time interval (mean firing frequency) or as the reciprocal of the interval between the first two action potentials during a spike train (initial firing frequency). For relatively short spike trains or for cells with little accommodation or antiaccommodation tendencies, the two plots may be similar.

The three cell types are compared in Figure 6, which gives $F-I$ plots for both initial frequency (panel $\mathrm{A}$ ) and mean frequency (panel B). The curves are generally sigmoidal in shape. The most striking difference is between the FS cells and the other two types (RS and LS). The FS cells can easily fire in excess of $100 \mathrm{~Hz}$, whereas the LS and RS cells have a maximum firing frequency of about $40 \mathrm{~Hz}$, and the slope of the $F-I$ relationship for FS cells is considerably steeper.

Differences among the four RS subtypes were relatively small for both initial and mean $F-I$ plots, so the illustrated graphs (Figures 6A and 6B) show data from just one subtype (RS3). On the other hand, there were appreciable differences among the four LS subtypes in terms of initial $F-I$ curves. The plots in Figure 6A show the two extreme subtypes (LS4 and LS1). The curves for the other two LS subtypes are intermediate between these extremes. Because there were negligible differences among the LS cells in the mean $F-I$ curves, only a single type (LS3) is plotted.

Delay versus input intensity relationships. A critical feature of the neuronal dynamics that enables temporal encoding is the relationship between input intensity $(I)$ and the delay $(D)$ from the input onset to the first spike. Recall that FS cells have the shortest delay, RS cells have a slightly longer delay, and LS cells have the longest delay. In our neurophysiological experiments, we most commonly saw delays in LS cells in the range of $1-3 \mathrm{sec}$. For all three model cell types, the delay is a monotonic decreasing function of the input intensity, as in our physiological data. The most striking difference is between the LS cells and the other two types (FS and RS). Substantial differences among both the RS subtypes and the LS 

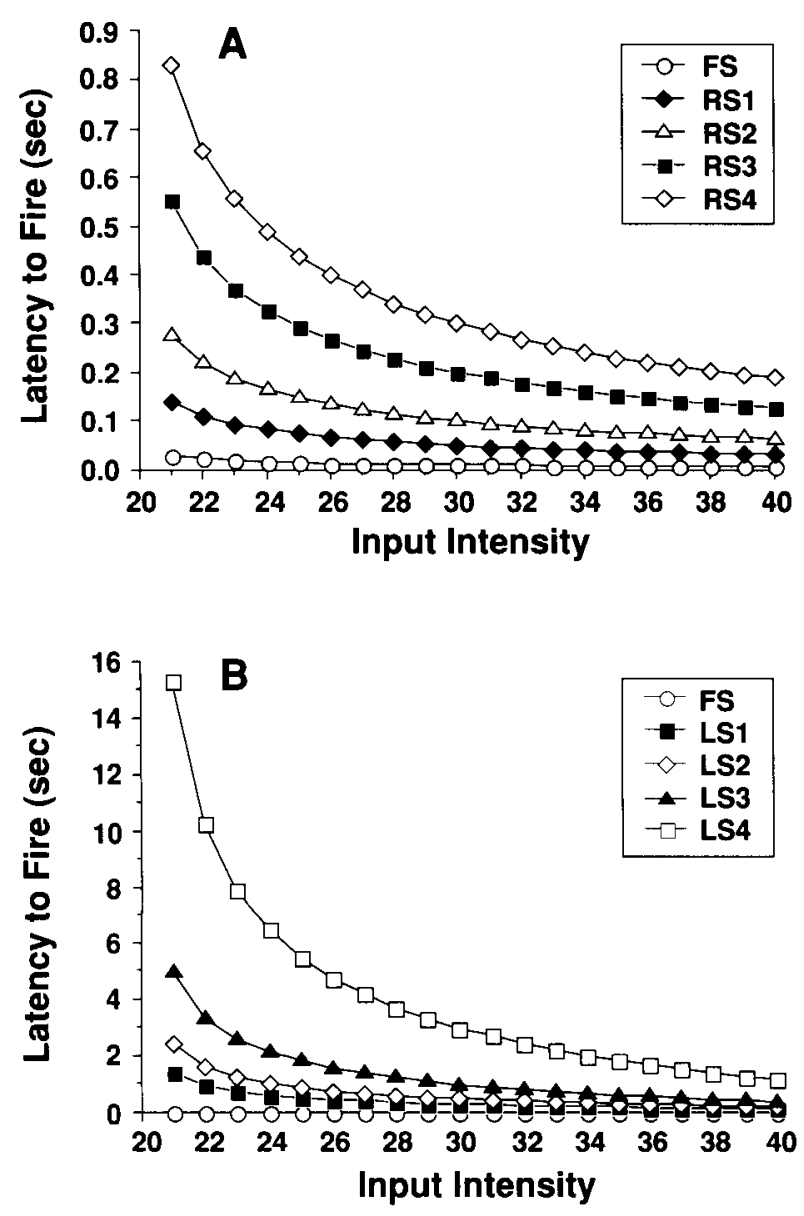

Figure 7. Delay to initiate spike firing as a function of input intensity. The relationship between delay $(D)$ and input intensity $(I)$ is shown for all three cell types. The $D-I$ curves are quite different for the various cell types and subtypes. (A) $D-I$ curves are compared for the four subtypes of regular-spiking (RS) cells (RS1-RS4), and these are contrasted with the fast-spiking (FS) cell as a common reference point. (B) $D-I$ curves are compared for the four subtypes of late-spiking (LS) cells (LS1-LS4), and these are contrasted with the FS cell. On this expanded time scale, the FS cell appears to fire with no delay, but in fact the delay is the same as it was when plotted in relationship to the RS cells. Note that the ordinate has been changed to include more time.

subtypes of our model cells are captured in the $D-I$ plots. Figure 7A shows $D-I$ curves for the four RS subtypes, as well as for the FS cell. Recall that the RS subtypes differ from each other only in regard to $\tau_{\mathrm{A}}$, which is smallest for RS1 neurons and largest for RS4 neurons (see Table E2 of Appendix E).

An analogous set of $D-I$ curves for LS and FS cells is shown in Figure 7B. As before, the FS cells are included as a common reference point. In contrast to the RS cells, LS subtypes all have the same time constant (equal to that for RS 1 cells) but differ with respect to an "inactivation" term (not included in FS or RS cells) that controls the delay for a given input. LSI cells exhibit the shortest delays, and LS4 cells produce the longest delays.
Interspike interval versus interval number relationships. The accommodation tendency exhibited by each type of cell is another critical aspect of the neuronal dynamics. Recall that FS cells are nonaccommodating, the RS cells are strongly accommodating, and the LS cells are anti-accommodating (see Figure 3). For our FS model neurons, the interspike interval is fixed across successive intervals, thus showing no accommodation. With stronger inputs, the interspike intervals are shorter (meaning higher firing frequency) but remain fixed within a spike train, given a constant input. For our RS model neurons, the interspike intervals across successive intervals are not constant in response to a fixed input but grow progressively longer until the cell stops firing. As the input intensity increases, the interspike intervals decrease (the firing frequency increases). The LS cells exhibit antiaccommodation, meaning that the interspike intervals become progressively shorter across a spike train. As the input intensity increases, the interspike intervals decrease (the firing frequency increases).

\section{Activity Delays and Windows}

One essential feature of the model is that chains of LS cells terminated by RS cells can produce a spectrum of activity windows delayed by various amounts from the CS onset. This spectrum of activity windows allows the mapping of time onto space, which makes it possible to use an ordinary Hebbian synaptic mechanism to learn long ISIs. An example of a partial set of activity windows is illustrated in Figure 8, where the abscissa is a continuous scale (time of firing from the CS onset) and the ordinate is a nominal scale (arbitrary cell identification number). Each horizontal set of circles represents the activity window for a particular cell; the individual circles indicate the points in time at which the associated neuron fired. Figure 8 illustrates the activity windows for 20 of the 189 neurons sending activity into the second layer of the ALa, where CS- and US-produced synaptic activity converge on the same set of postsynaptic cells.

For visual convenience, the windows are arranged in ascending order according to delays from the CS onset. Synaptic inputs to the second layer of the ALa, whose activity can overlap the US input, are subject to modification by the Hebbian learning rule. Greater overlap produces a faster rate of synaptic modification. The mean $( \pm S D)$ duration of the 189 activity windows, calculated from seven noise-injected trials, was $778 \pm 185 \mathrm{msec}$. With more neurons to form longer chains or recurrent circuitry, it is possible to encode (by mapping time onto space) a much larger range of CS-US intervals (see the Discussion section).

\section{Pairing-Specific Temporal Encoding}

Prior to conditioning, all second-layer ALa cells were unresponsive to the CS. In the experimental condition, after six conditioning trials in which the CS and US were explicitly paired (so as to overlap temporally), there was always CS-produced output, regardless of the ISI used 


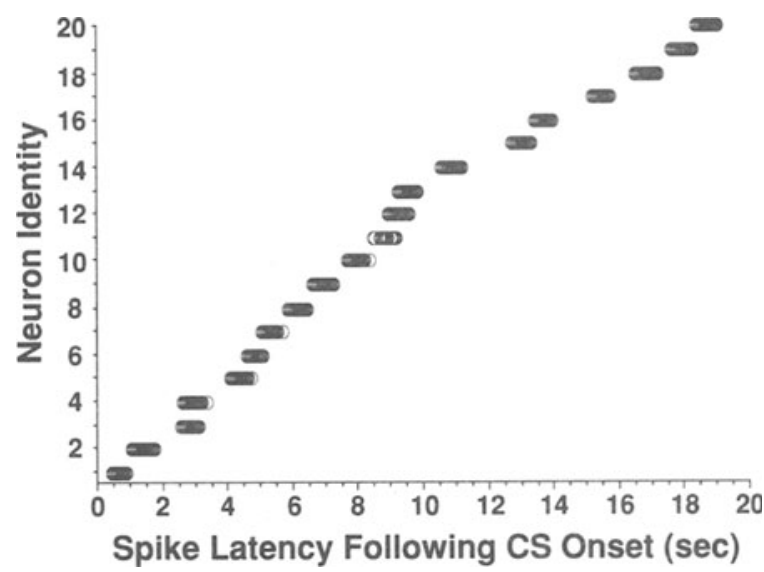

Figure 8. Spectrum of delayed firing onsets and associated activity windows. Each horizontal line is the time over which a particular cell in the adjacent lateral amygdala (ALa) was active, relative to the onset of the conditioned stimulus (CS). These are the first-layer ALa neurons whose axonal projections make Hebbian synapses onto second-layer ALa neurons, which also receive a direct input from the unconditioned stimulus. The activity windows are arranged from bottom to top in order of increasing delays. The cell whose activity is illustrated in the lower left began firing soon after the CS onset and stopped firing long before the CS terminated. The cell whose activity is shown in the upper right began firing long after the CS onset. Illustrated here is a sample of just 20 of the 189 ALa neurons that participate in time-space mapping (see the text for further explanation).

in conditioning. In contrast, in the control condition, in which the CS and US were explicitly unpaired (so that there was no temporal overlap between them), six trials never resulted in CS-produced output (even when noise was added; see below), because synaptic potentiation could not occur. Thus, any CS-produced output from the ALa is always and exclusively the result of associative learning. In what follows, therefore, we do not make explicit comparisons with the control (pseudoconditioning) group, because such comparisons furnish no additional information. This demonstration of pairing-specific output was, of course, a minimal requirement for the model.

In regard to conditioning, the goals of this research were more ambitious and concerned temporal aspects of the learning. Recall that 13 different ISI training intervals were explored in the experimental condition (paired CSUS). The first question was whether, in this very simplified model, the circuit could encode the CS-US interval, using only properties suggested by the anatomy and physiology. In addressing this issue, the network was trained using noise-free synapses. We then used a testing trial (CS alone) to examine the relationship between the training ISI (on the abscissa) and the latency of all CSgenerated output activity in second-layer ALa neurons (on the ordinate).

Each point in the scatterplot shown in Figure 9A indicates the firing latency (from the CS onset) of second-layer ALa neurons as a function of the training ISI. The results plotted at each ISI represent the consequence of six con- ditioning trials (CS-US pairings). The best-fitting regression line to describe these data $(\tilde{y}=m x+b)$, based on the method of least squares (see Figure 9A), had a slope $m$ close to unity $(m=0.995)$ and a $y$-intercept slightly greater than zero $(b=0.027 \mathrm{sec})$. The excellent temporal encoding was reflected in the coefficient of determination, $r^{2}=.996$. The answer to our first question was that the circuit clearly is able to learn CS-US intervals in the range of $0.5-16 \mathrm{sec}$ and to do so with impressive precision. ${ }^{3}$

\section{Appropriate Noise Causes \\ Weber-Like Temporal Encoding}

The previous result naturally leads to our second question, which was whether the temporal learning mechanism naturally conforms to a Weber-like law. A modern interpretation of Weber's law (Weber, 1851) - in the context of the present experiments-is that variability in the response latency $(\sigma)$ associated with each training ISI should be proportional to the mean response latency $(\mu)$ associated with that ISI (Gibbon, 1977; Gibbon et al., 1997). That is, the coefficient of variation ( $c v)$ should be relatively constant across ISIs ( $c \nu=\sigma / \mu=$ constant). There is a sizable body of data in support of this generalization (Gibbon, 1977). In stark contrast to this expectation, what we observed in noise-free simulations (Figure $9 \mathrm{~A}$ ) was that the response latency variability was roughly uniform and independent of the mean response latency. Consequently, the $c v$ decreased monotonically with increasing ISIs.

One hypothesis is that the Weber law (for time) results from underlying random variation in a noise variable (Gibbon et al., 1997). As indicated earlier, there are several likely noise sources in regard to CS processing in the PRALa circuit. The third question we addressed was whether adding noise to the model would result in Weber-like temporal learning. In a second set of simulations, we added noise to the synapses by sampling a new Gaussian variate at every 1-msec time step (see Equation 24 of Appendix D). Even with relatively large amounts of synaptic noise, the response latency variability did not increase with longer training ISIs. The results (not shown) resembled the noise-free simulations shown in Figure 9A. Clearly, noise per se was not sufficient to generate the Weber effect.

A third set of simulations revealed an important interaction between the noise frequency spectrum and the internal system dynamics. In these simulations, lower frequency noise was introduced by randomly sampling from a uniform distribution (with limits of 50 and 250 time steps) the time step on which the random variate would be changed to a new value. The set of conditioning sessions given, and the number of trials within each conditioning session, remained the same as in the noise-free simulations. Under these circumstances, even relatively small amounts of this low-frequency noise produced Weber-like encoding (Figure 9B); specifically, the response variability increased with the mean response latency (and the 

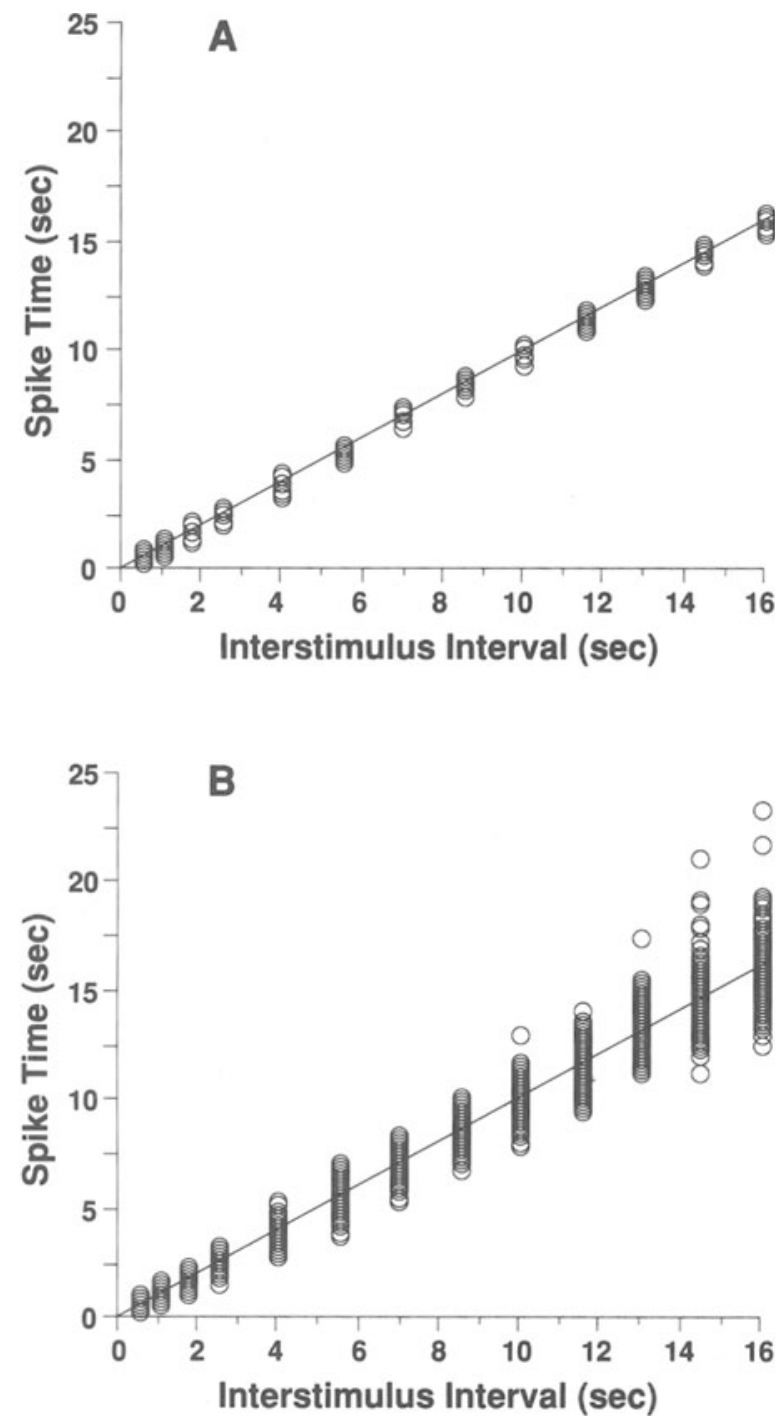

Figure 9. Simulated Pavlovian conditioning demonstrating accurate temporal learning. The conditioned stimulus (CS) fires cells in the second layer of the lateral amygdala after conditioning but not before, and only if the $C S$ and the unconditioned stimulus (US) are explicitly paired (see the text). (A) The scatterplot reveals excellent temporal encoding of the CS-US interval. Note that the accuracy of predicting the time of the US onset does not decrease as the interstimulus interval (ISI) increases, in contrast to expectations of a Weber-like encoding principle. (B) When the synapses were injected with low-frequency noise, the response latency variance clearly increased with the mean response latency and the training ISI. The results are the sum of seven sets of simulations for each ISI. Further analysis indicated that the noise-produced latency variability increased linearly with the mean response latency, meaning that the coefficient of variation exhibited no trend and little variability across different ISI conditioning intervals. The earliest spikes always preceded the US onset.

training ISI). The results shown in Figure 9B are for the case in which the magnitude of the noise, $\kappa_{c v}$, was relatively small ( $\kappa_{c v}$ was drawn from a uniform distribution with limits of 0.000 and 0.100 ).
Note also that the accuracy of the temporal encoding remained excellent. Over the training range of $0.5-16 \mathrm{sec}$, the best-fitting straight line $(\tilde{y}=m x+b)$, based on the method of least squares (see Figure 9B), had a slope $m$ near unity $(m=1.008)$ and a $y$-intercept slightly greater than zero $(b=0.077)$. The excellent temporal encoding was reflected in the coefficient of determination $r^{2}=.971$. The earliest output spikes always anticipated the US onset, an effect that we have just begun to study (Faulkner, McGann, et al., 1997).

\section{Two Sources of Variability in Response Latency for Each ISI}

Examination of the data (Figure 9) reveals two sources of variability in the conditioned response latency for a given ISI. The first is associated with the intrinsic encoding accuracy of the circuit, based on factors under our control. This small source of variability was evident in the noise-free simulations (Figure 9A). It clearly violated the Weber law, in that the $c v$ was not constant but decreased monotonically with larger conditioning ISIs; that is, the intrinsic or noise-free source of variability was roughly constant across the range of training ISIs. With greater numbers of neurons and smaller activity windows, this source of variability can be made arbitrarily small.

To isolate the contributions of noise per se to the response variability, we subtracted the intrinsic variability (evident in Figure 9A) from the total variability after noise injection (the variability increase evident in Figure 9B) as follows. We calculated the variance associated with the intrinsic (noise-free) circuit function for each ISI, then subtracted it from the total variance for each ISI associated with the simulations that included noise injection. The remaining variance was then used as our estimate of the noise-produced latency variance. From the square root of this remaining variance and the mean response latency associated with simulations that included noise, we found the noise-produced $c v$ at each ISI. The regression of $c v$ on ISI revealed that the noise-produced $c v$ was essentially constant and independent of the ISI. For the best fitting regression line $(\tilde{y}=m x+b)$, the slope was essentially zero $\left(m=-4.872 \cdot 10^{-7}\right)$, and the $y$ intercept $(b=0.083)$ was close to the mean value $(\overline{c v}=$ 0.080 ). Thus, injection of appropriate noise produced a Weber-like relationship between error and magnitude.

\section{DISCUSSION}

A fundamental problem in understanding temporal aspects of learning has been to identify possible cellular and circuit-level neurobiological mechanisms that can be utilized across the range of tenths of seconds to tens of seconds (Gibbon et al., 1997). A common assumption is that this is a circuit-level problem that can be solved simply by invoking notions of reverberatory loops. Unfortunately, such loops become unrealistically large with conventional neuronal dynamics (RS cells). The numbers of neurons and/or the numbers of reverberations are so large that the circuit must become unstable and easily dis- 
ruptable - even if one assumes an implausibly precise topographical projection system around the loop.

By contrast, using a combination of RS and LS cells, we have shown that purely feedforward chains of no more than 16 neurons (14 in the PR and 2 in the ALa) can accurately encode time intervals from 0.5 to $16 \mathrm{sec}$, and this occurs with no assumptions regarding the precision of projections beyond what is seen experimentally. The methods and results presented here are the first step in formalizing an earlier qualitative model (Faulkner, 1997; Faulkner, Tieu, \& Brown, 1997) that emphasized the role of the PR-ALa region in temporal aspects of fear conditioning. This has been a bottom-up approach, starting with experimentally observed neuronal properties and probable connections. To implement the computational model, we have been developing efficient computer code that is able to simulate sizable amounts of real time in circuits containing large numbers $(\sim 1,600)$ of dynamically realistic neurons (Faulkner, McGann, et al., 1997; Tieu et al., 1996).

\section{Solution to the Contiguity Dilemma}

For centuries, philosophers and psychologists have focused on temporal contiguity as the kernel of associative learning. With the discovery of associative LTP and its underlying Hebbian mechanism, it became clear that a cellular mechanism exists for contiguity-based learning (Barrionuevo \& Brown, 1983; T. H. Brown et al., 1990; Kelso \& Brown, 1986; Kelso et al., 1986). Indeed, some forms of perceptual learning are undoubtedly based on contiguity. Although it is certain that humans and other animals do form associations among contiguous events, it is equally clear that they also learn temporal relationships among sequential events. The problem is that the properties of associative LTP do not furnish insight into the neurobiological basis of temporal aspects of learning (Shors \& Matzel, 1997). This fact has been invoked to question whether associative LTP even has the right properties for certain forms of learning (Shors \& Matzel, 1997).

By creating a spectrum of activity windows (Figure 8) that map time onto space, the model is able to learn sequential temporal relationships using an ordinary Hebbian synaptic mechanism. We outlined a general solution, tested it through computer simulations of a dynamical neuronal circuit, and showed that the circuit was able to encode and learn time intervals ranging from tenths of a second to tens of seconds (Figure 9). The intrinsic neurodynamics and cellular neuroanatomy were the key to this temporal learning mechanism. The existence of LS cells that (1) can delay firing for seconds, (2) exhibit antiaccommodation, and (3) are organized into chains furnishes a general approach for encoding large time intervals. We do not assume that the mechanisms described here are unique to fear conditioning circuitry. They may be involved more generally in temporal aspects of behavior that involve many other brain regions with which the perirhinal-amygdala (PR-AM) region has connections, including the frontal lobes and basal ganglia.

\section{Conditions and Accuracy of Associative Learning}

In these simulations, learning reliably occurred in the ALa when the CS was explicitly paired with (temporally overlapped) the US. Most importantly, the circuit not only acquired a neural conditioned response (CR), but the timing of the CR matched the training ISI. Temporal learning was immediately retrievable (and implicitly represented) by the latency of CS-produced activity in the output cells (the "neural CR"), which began just before the expected time of the US onset. Interestingly, the temporal encoding was highly accurate (the coefficient of determination remained high, $r^{2}>.95$, even after the introduction of noise into the circuitry), as measured by the relationship between the CS-US training ISI used during conditioning and the latency of CS-produced activity during testing (Figure 9B). There are obvious and easy ways to extend the time domain over which the model can perform. Because such extensions increase simulation times but do not introduce new principles, we limited the upper end of the ISIs that could be encoded to about one fourth of a minute. The upper limit can be extended by using longer chains of LS cells and/or by introducing activity loops.

As expected on the basis of the circuitry, learning did not occur when the CS and US were explicitly unpaired in the pseudoconditioning regime. Perhaps not so obvious is the fact that the circuit as constructed also does not allow trace conditioning. Although this is not difficult to incorporate into the computational model, to do so requires adding recurrent excitatory connections, which we wanted to avoid initially for simplicity in favor of a purely feedforward network. The effects of recurrent circuitry, included in our conceptual model (Faulkner, Tieu, \& Brown, 1997), are discussed below in connection with other learning phenomena that we are currently exploring.

\section{Weber Effect and Noise Spectrum}

In the absence of added noise, the accuracy of temporal encoding does not decrease as the CS-US interval increases (Figure 9A). This is a good feature from an engineering point of view, but it does not comply with what is known from the behavioral literature. A modern interpretation (Gibbon et al., 1997) of the Weber law (Weber, 1851 ) in the time domain is that the coefficient of variation of the response should be proportional to the mean response latency, which is clearly not true in Figure 9A. However, the results were quite different when we added the appropriate noise (Figure 9B) and were consistent with the hypothesis (Gibbon et al., 1997) that temporal aspects of the Weber law can emerge from random noise.

It is interesting to note that the spectral aspects of the noise are critical in producing the Weber effect when it emerges within the intrinsic system neurodynamics. High- 
frequency noise was essentially without effect, because these model neurons are dynamically realistic in acting as low-pass filters. When the noise spectrum became appropriate to the system dynamics, the Weber effect immediately emerged in remarkable form. We conclude that there is an interaction between the spectral/amplitude aspects of the noise and the neuron/circuit dynamics, a phenomenon that warrants further investigation at the neuronal and behavioral levels. This is a good example of the kind of phenomenon that cannot emerge from learning models that ignore the underlying neurodynamics. Interestingly, the $c v$ was somewhat less than 0.1 , which is of the magnitude reported by many behavioral studies (see Figure 3 of Gibbon et al., 1997).

\section{Probable Effects of Adding Recurrent Synapses}

Although the computational model is a purely feedforward circuit (Figure 5), it is important to recall that the amygdala and $P R$ are replete with intrinsic recurrents, reciprocal connections with each other, and loops that include other brain regions. Introducing excitatory feedback into a network of dynamical neurons invites instability, which is why it was not done in this first model; but it is worth noting that even relatively small loops that include LS cells can encode very large amounts of time. The anatomy is consistent with the possibility that chains and loops could exist within both layer II/III and layer VI (Figure 1A), and there is also evidence that these two regions may themselves be reciprocally connected by LS cells (Faulkner \& Brown, in press).

Adding recurrent excitation to the circuitry requires also adding appropriate feedforward and feedback inhibition (to prevent epileptiform activity). Indeed these brain regions are well known to be very seizure prone. We are currently developing models with recurrent excitation to explore a variety of other computations that may be supported by the PR. It seems reasonable to regard small loops of LS cells as a robust way to instantiate stimulus traces (Schmajuk, 1997) that persist beyond the duration of the initiating event. By making the neural activity outlast the termination of the initiating stimulus (see Young, Otto, Fox, \& Eichenbaum, 1997), it becomes possible to span gaps in time. This is important in trace Pavlovian conditioning paradigms and also in delayed nonmatching-to-sample tasks. There has also been a long history of interest in recurrent circuits functioning as autoassociators (see Anderson, 1985; Hinton \& Anderson, 1981; Hopfield, 1984; Kohonen, 1984). In reviewing possible autoassociative functions of the hippocampus, this lab previously noted that

Temporal sequences of patterns can be learned by an autoassociator if the output is fed back, after a time delay, into the input (Kohonen, 1984). Increasing the number of different time delays can improve the temporal learning. The spatiotemporal pattern completion that such a network can perform is reminiscent of what some classical learning theorists used to call redintegration (Hull, 1929). Redin- tegration was once considered a fundamental mental process (Hollingworth, 1928), but until recently it was not clear how this might be implemented neurophysiologically. (T.H. Brown \& Zador, 1990, p. 387)

We should emphasize that autoassociative recurrent circuits can use what could be termed contextual information as a retrieval cue (Anderson, 1985; Anderson, 1995; Churchland, 1995; Hinton \& Anderson, 1981; Hopfield, 1984; Kohonen, 1984). This may, in fact, be one possible way that context enters into the expression of conditioning (Bouton \& Bolles, 1985). In such recurrent circuits, context can also be used to bind episodic information across time (for a recent discussion, see Wallenstein, Eichenbaum, \& Hasselmo, 1998). As more is learned about the microcircuitry and plasticity of the PR-AM system, it will clearly be important to explore the contributions of recurrent circuitry to contextual and trace Pavlovian fear conditioning.

\section{Relationships to Certain Other Timing Models}

A distinction is sometimes made between networks that encode time on the basis of a spectrum of delay lines and those that encode time on the basis of intrinsically timevarying processes (Anton, Lynch, \& Granger, 1991; Bankes \& Margoliash, 1993; Braitenberg, 1967; Buonomano \& Merzenich, 1995; Desmond \& Moore, 1988; Fujita, 1982; Hopfield, 1995; Jaffe, 1992; Jeffress, 1948; Mial, 1989; Moore et al., 1989; Sullivan, 1982). Our solution incorporates both approaches by making use of realistic endogenous neuronal dynamics in an appropriate circuit to create a spectrum of time-delayed activity windows that can accurately encode temporal information over the range of tenths of seconds to many seconds.

Our computational model can be seen as a specific, neurobiologically based platform for more abstract ideas that have been expressed and utilized elsewhere. The VET mode ${ }^{4}$ of Moore and colleagues (Moore \& Choi, 1997) is a good example. Their "cascades of spreading activation" are described as follows:

This spreading activation sequentially engages timetagged "input elements." These input elements can be regarded as serial components of the nominal CS. . . Computations on these sequentially activated elements give rise to CRs that mirror the CS-US intervals employed in training. Such CRs are said to be temporally adaptive. ... Both CS onsets and offsets are presumed to trigger cascades of spreading activation. (Moore \& Choi, 1997, p. 119)

In our model, serial components of the nominal CS are obtained via a spectrum of delay lines and associated activity windows (Figures 5 and 8 ). One important difference is that, in our purely excitatory and feedforward simulated circuit (Figure 5), activity is only initiated by the CS onset. The idea of activity windows also appears in the Mauk-Donegan model (Mauk \& Donegan, 1997), although their windows have different neurobiological origins and computational implications. 


\section{Biophysical and Molecular Mechanisms}

The ability to engulf large amounts of time with single LS neurons results from the unusual manner in which they delay firing. Their long latency to fire is not due to a long membrane time constant. In fact, the membrane time constants of these cells are within the normal range for the PR (Faulkner, 1997; Faulkner \& Brown, 1996). Preliminary voltage-clamp studies (Faulkner, 1997) are consistent with the possibility that the delayed firing is due to a potassium current that (1) is strongly activated near the spike threshold and (2) inactivates unusually slowly. This may be what has been termed a D-current (Johnston \& Wu, 1995). There are a large number of potassium currents that have various inactivation properties. As more is learned about the biophysics and molecular biology of potassium channels (see Edwards \& Weston, 1997; Hoshi, Zagotta, \& Aldrich, 1991; Johnston \& Wu, 1995; Lopez-Barneo, Hoshi, Heinemann, \& Aldrich, 1993), it should become possible to identify with some confidence the conductance mechanisms responsible for delayed spiking. Such an identification would be a fascinating first step in formulating a possible linkage between conductance properties of a particular class of ion channels and a fundamental aspect of cognitive and motor function.

\section{Circuit-Level Formalism for Bridging Levels of Analysis}

The present simulator (YNET) preserves the dynamical input-output properties of the neurons without incurring the overhead associated with traditional, conductancebased compartmental modeling, which this laboratory has been doing since the late 1970 s. Further improvements in this intermediate-level simulator will allow easy transitions between connectionist and biophysical studies and will enable a deeper understanding of the nature and extent to which fundamental computations of large circuits emerge from dynamical aspects of the individual neurons. We suspect that it will be necessary to explore relatively large-scale and dynamiçally realistic circuits to begin generating deep theoretical insights into the mechanisms of learning in parallel-distributed systems. This entails incorporating, in a parallel-distributed fashion, the contextual/temporal framework within which motor and cognitive aspects of learning mechanisms operate (see Bouton, 1993; Bouton \& Bolles, 1985; Church, 1984; Gibbon et al., 1997; Holland, 1986; Ivry, 1996; Rescorla, Durlach, \& Grau, 1985; Schmajuk, 1997; Wallenstein et al., 1998).

\section{REFERENCES}

AggLeton, J. P. (1992). The amygdala: Neurobiological aspects of emotion, memory and mental dysfunction. New York: Wiley-Liss. ANDERSON, J. A. (1985). What Hebb synapses build. In W. B. Levy, J. A. Anderson, \& S. Lehmkuhle (Eds.), Synaptic modification, neuron selectivity and nervous system organization (pp. 153-174). Hillsdale, NJ: Erlbaum.
ANDERSON, J. A. (1995). An introduction to neural networks. Cambridge, MA: MIT Press.

ANTON, P. S., LYNCH, G., \& GRANGER, R. (1991). Computation of frequency-to-spatial transform by olfactory bulb glomeruli. Biological Cybernetics, 65, 407-414.

Applegate, C. D., Frysinger, R. C., Kapp, B. S., \& Gallagher, M. (1982). Multiple unit activity recorded from amygdala central N. during Pavlovian heart rate conditioning in rabbit. Brain Research, 238, 457-462.

Applegate, C. D., Kapp, B. S., Underwood, M. D., \& MCNall, C. L. (1983). Autonomic and somatomotor effects of amygdala central nucleus stimulation in awake rabbits. Physiology \& Behavior, 31, 353360.

Armony, J. L., Servan-Schreiber, D., Cohen, J. D., \& LeDoux, J. E. (1995). An anatomically constrained neural network model of fear conditioning. Behavioral Neuroscience, 109-246-257.

Bankes, S. C., \& Margoliash, D. (1993). Parametric modeling of the temporal dynamics of neuronal responses using connectionist architectures. Journal of Neurophysiology, 69, 980-991.

Barrionuevo, G., \& Brown, T. H. (1983). Associative long-term synaptic potentiation in hippocampal slices. Proceedings of the $\mathrm{Na}$ tional Academy of Sciences, 80, 7347-7351.

Bear, M. F., \& Abraham, W. C. (1996). Long-term depression in hippocampus. Annual Review of Neuroscience, 19, 437-462.

BEAR, M. F., \& MALENKA, R. C. (1994). Synaptic plasticity: LTP and LTD. Current Opinion in Neurobiology, 4, 389-399.

BegGs, J. M., MOYER, J. R., \& BRowN, T. H. (1997). Synaptic integration in perirhinal delay neurons. Society for Neuroscience Abstracts, 23, 655 .

Bienenstock, E. L., Cooper, L. N., \& Monro, P. W. (1982). Theory for the development of neuron selectivity: Orientation specificity and binocular interaction in visual cortex. Journal of Neuroscience, 2, 32 48.

Bordi, F., LeDoux, J., Clugnet, M. C., \& Pavlides, C. (1993). Singleunit activity in the lateral nucleus of the amygdala and overlying areas of the striatum in freely behaving rats: Rates, discharge patterns, and responses to acoustic stimuli. Bchavioral Neuroscience, 107, 757 769 .

Bouton, M. E. (1993). Context, time, and memory retrieval in the interference paradigms of Pavlovian conditioning. Psychological Bulletin, 114, 80-99.

Bouton, M. E., \& Bolles, R. C. (1985). Contexts, event-memories, and extinction. In P. D. Balsom \& A. Tomie (Eds.), Context and learning (pp. 133-166). Hillsdale, NJ: Erlbaum.

BRAITENBERG, V. (1967). Is the cerebellar cortex a biological clock in the millisecond range? Progress in Brain Research, 25, 334-346.

Brown, B. L., Hemmes, N. S., \& Cabeza de Vaca, S. (1992). Effects of intratrial stimulus change on fixed-interval performance: The roles of clock and memory processes. Animal Learning \& Behavior, 20, 83-93.

Brown, J. S., Kalish, H. I., \& Farber, I. E. (1951). Conditioned fear as revealed by magnitude of startle response to an auditory stimulus. Journal of Experimental Psychology, 41, 317-328.

Brown, T. H., Chapman, P. F., Kairiss, E. W., \& Keenan, C. L. (1988) Long-term synaptic potentiation. Science, 242, 724-728.

Brown, T. H., \& FAULKNER, B. (1998). Hebbian synapses. Encyclopedia of neuroscience (CD ROM ed.). Amsterdam: Elsevier.

Brown, T. H., Ganong, A. H., Kairiss, E. W., Keenan, C. L., \& KELSO, S. R. (1989). Long-term potentiation in two synaptic systems of the hippocampal brain slice. In J. H. Byrne \& W. O. Berry (Eds.), Neural models of plasticity: Experimental and theoretical approaches (pp. 266-306). San Diego: Academic Press.

Brown, T. H., Kairiss, E. W., \& Keenan, C. L. (1990). Hebbian synapses: Biophysical mechanisms and algorithms. Annual Review of Neuroscience, 13, 475-511.

Brown, T. H., \& Zador, A. M. (1990). Hippocampus. In G. M. Shepherd (Ed.), The synaptic organization of the brain (3rd ed., pp. 346388). Oxford: Oxford University Press.

Buonomano, D. V., \& MaUK, M. D. (1994). Neural network model of 
the cerebellum: Temporal discrimination and the timing of motor responses. Neural Computation, 6, 38-55.

Buonomano, D. V., \& MERZENich, M. M. (1995). Temporal information transformed into a spatial code by a neural network with realistic properties. Science, 267, 1028-1030.

Burwell, R. D., \& Amaral, D. G. (1998). Perirhinal and postrhinal cortices of the rat: Interconnectivity and connections with the entorhinal cortex. Journal of Comparative Neurology, 391, 293-321.

Burwell, R. D., Witter, M. P., \& Amaral, D. G. (1995). Perirhinal and postrhinal cortices of the rat: A review of the neuroanatomical literature and comparison with findings from the monkey brain. Hippocampus, 5, 390-408.

Cahill, L., \& McGaugh, J. L. (1998). Mechanisms of emotional arousal and lasting declarative memory. Trends in Neurosciences, 21 , 294-299.

CANLI, T., \& Brown, T. H. (1996). Amygdala stimulation enhances the rat eyeblink reflex through a short-latency mechanism. Behavioral Neuroscience, 110, 51-59.

CARR, C. E., \& KoNISHI, M. (1988). Axonal delay lines for time measurement in the owl's brainstem. Proceedings of the National Academy of Sciences, 85, 8311-8315.

Cavus, L., \& TeYler, T. (1996). Two forms of long-term potentiation in area CA1 activate different signal cascades. Journal of Neurophysiology, 76, 3038-3047.

Chapman, P. F., Kairiss, E. W., Keenan, C. L., \& Brown, T. H. (1990). Long-term synaptic potentiation in the amygdala. Synapse, 6, 271278.

CHURch, R. M. (1984). Properties of the internal clock. In J. Gibbon \& L. G. Allan (Eds.), Timing and time perception (Annals of the New York Academy of Sciences, Vol. 423, pp. 566-582). New York: New York Academy of Sciences.

Church, R. M., \& Broadbent, H. A. (1991). A connectionistic model of timing. In M. L. Commons, S. Grossberg, \& J. E. R. Staddon (Eds.), Neural network models of conditioning and action (pp. 225240). Hillsdale, NJ: Erlbaum.

Churchland, P. M. (1995). The engine of reason, the seat of the soul. Cambridge, MA: MIT Press.

Corodimas, K. P., \& LeDoux, J. (1995). Disruptive effects of posttraining perirhinal cortex lesions on conditioned fear: Contributions of contextual cues. Behavioral Neuroscience, 109, 613-619.

Davis, M., Schlesinger, L. S., \& Sorenson, C. S. (1989). Temporal specificity of fear conditioning: Effects of different conditioned stimulus-unconditioned stimulus intervals on the fear-potentiated startle effect. Journal of Experimental Psychology: Animal Behavior Processes, 15, 295-310.

Deacon, T. W., Eichenbaum, H., Rosenberg, P., \& Eckmann, K. W. (1983). Afferent connections of the perirhinal cortex in the rat. Journal of Comparative Neurology, 220, 168-190.

Debanne, D., Gähwiler, B. H., \& Thompson, S. M. (1994). Asynchronous pre- and postsynaptic activity induces associative longterm depression in area $\mathrm{CA} 1$ of the rat hippocampus in vitro. Proceedings of the National Academy of Sciences, 91, 1148-1152.

DESMOND, J. E. (1990). Temporally adaptive responses in neural models: The stimulus trace. In M. Gabriel \& J. Moore (Eds.), Learning and computational neuroscience: Foundations of adaptive networks (pp. 421-461). Cambridge, MA: MIT Press.

DESMOND, J. E., \& MOORE, J. W. (1988). Adaptive timing in neural networks: The conditioned response. Biological Cybernetics, 58, 405-415.

EDWARDS, G., \& Weston, A. H. (1997). Voltage-gated ion channels. In R. W. Davies \& B. J. Morris (Eds.), Molecular biology of the neuron (pp. 145-175). Glasgow: Bios Scientific Publishers.

Falls, W. A., Bakken, K. T., \& Heldt, S. A. (1997). Lesions of the perirhinal cortex interfere with conditioned excitation but not with conditioned inhibition of fear. Behavioral Neuroscience, 111, 476486.

Fanselow, M. S. (1998). Pavlovian conditioning, negative feedback, and blocking: Mechanisms that regulate association formation. Neuron, 20, 625-627.

Fanselow, M. S., \& Kim, J. J. (1994). Acquisition of contextual Pavlovian fear conditioning is blocked by application of an NMDA receptor antagonist D,L-2-amino-5-phosphonovaleric acid to the basolateral amygdala. Behavioral Neumscience, 108, 210-212.
FAULKNER, B. (1997). Amygdala-perirhinal memory system: Synapses, neurons, and circuits. Unpublished doctoral dissertation, Yale University, New Haven.

FAULKNER, B., \& Brown, T. H. (1995). Physiology and morphology of perirhinal cortical neurons. Society for Neuroscience Abstracts, 21, 596.

FAUlKNER, B., \& Brown, T. H. (1996). Spatial organization and diversity of neurons in the rat perirhinal-lateral amygdala region. Society for Neuroscience Abstracts, 22, 1742.

FAULKNER, B., \& Brown, T. H. (in press). Morphology and physiology of neurons in the rat perirhinal-lateral amygdala area. Journal of Comparative Neurology.

Faulkner, B., McGann, J. P., Tieu, K. H., \& Brown, T. H. (1997). Perirhinal-amygdala circuit model accounts for key temporal aspects of Pavlovian conditioning. Society for Neuroscience Abstracts, 23, 655.

Faulkner, B., Tieu, K. H., \& Brown, T. H. (1997). Mechanism for temporal encoding in fear conditioning: Delay lines in perirhinal cortex and lateral amygdala. In J. Bower (Ed.), Computational neuroscience: Trends in research 1997 (pp. 641-645). New York: Plenum.

FujITA, M. (1982). An adaptive filter model of the cerebellum. Biological Cybernetics, 45, 195-206.

Gallagher, M., \& Chiba, A. N. (1996). The amygdala and emotion. Current Opinion in Neurobiology, 6, 221-227.

Gallagher, M., Kapp, B. S., McNall, C. L., \& Pascoe, J. P. (1981). Opiate effects in the amygdala central nucleus on heart rate conditioning in rabbits. Pharmacology, Biochemistry \& Behavior, 14, 497505.

GibBon, J. (1977). Scalar expectancy theory and Weber's law in animal timing. Psychological Review, 84, 279-325.

Gibbon, J., Malapani, C., Dale, C. L., \& Gallistel, C. R. (1997). Toward a neurobiology of temporal cognition: Advances and challenges. Current Opinion in Neurobiology, 7, 170-184.

Gluck, M. A., ReIfSNider, E. S., \& Thompson, R. F. (1990). Adaptive signal processing in the cerebellum: Models of classical conditioning and VOR adaptation. In M. A. Gluck \& D. E. Rumelhart (Eds.), Neuroscience and connectionist theory (pp. 131-186). Hillsdale, NJ: Erlbaum.

Gormezano, I., Prokasy, W. F., \& Thompson, R. F. (Eds.) (1987). Classical conditioning ( $3 \mathrm{rd}$ ed.). Hillsdale, NJ: Erlbaum.

GrossberG, S., \& SchmajuK, N. A. (1989). Neural dynamics of adaptive timing and temporal discrimination during associative learning. Neural Networks, 2, 79-102.

Grover, L. M., \& TeYLer, T. J. (1990). Two components of LTP induced by different patterns of afferent activation. Nature, 347, 477-479.

Harrington, D. L., \& HaAland, K. Y. (1998). Sequencing and timing operations of the basal ganglia. In D. A. Rosenbaum \& C. E. Collyer (Eds.), Timing of behavior: Neural, psychological, and computational perspectives (pp. 35-61). Cambridge, MA: MIT Press.

Heynen, A. J., Abraham, W. C., \& Bear, M. F. (1996). Bidirectional modification of CAl synapses in the adult hippocampus in vivo. $\mathrm{Na}$ ture, 381, 163-166.

Hinton, G., \& Anderson, J. A. (1981). Parallel models of associative memory. Hillsdale, NJ: Erlbaum.

Holland, P. C. (1986). Temporal determinants of occasion setting in feature-positive discriminations. Animal Learning \& Behavior, 14, 111-120.

Hollingworth, H. L. (1928). Psychology: Its facts and principles New York: D. Appleton.

Hopfield, J. J. (1984). Neurons with graded response have collective computational properties like those of two-state neurons. Proceedings of the National Academy of Sciences, 81, 3088-3092.

HOPFIELD, J. J. (1995). Pattern recognition computation using action potential timing for stimulus representation. Nature, 376, 33-36.

Hoshi, T., ZagotTa, W. N., \& Aldrich, R. W. (1991). Two types of inactivation in Shaker $\mathrm{K}+$ channels: Effects of alterations in the carboxy-terminal region. Neuron, 7, 547-556.

HuA, S. E., \& Houk, J. C. (1997). Cerebellar guidance of premotor network development and sensorimotor learning. Learning \& Memory, 4, 63-76.

HuLL, C. L. (1929). A functional interpretation of the conditioned reflex. Psychological Review, 36, 498-511. 
Inagaki, S., Matsuda, Y., Nakai, Y., \& Takagi, H. (1990). Calcitonin gene-related peptide (CGRP) immunoreactivity in the afferents to the caudate-putamen and perirhinal cortex of rats. Brain Research, 537, 263-270.

IVRY, R. B. (1996). The representation of temporal information in perception and motor control. Current Opinion in Neurobiology, 6, 851857.

JAFFE, S. (1992). A neuronal model for variable response latency. In F. H. Eeckman (Ed.), Analysis and modeling of neural systems (pp. 405-410). Boston: Kluver.

JEFFRESS, L. A. (1948). A place theory of sound localization. Journal of Comparative and Physiological Psychology, 41, 35-39.

Johnston, D., \& WU, S. M.-S. (1995). Foundations of cellular neurophysiology. Cambridge, MA: MIT Press.

KapP, B. S., Frysinger, R. C., Gallagher, M., \& Haselton, J. R. (1979). Amygdala central nucleus lesions: Effect on heart rate conditioning in the rabbit. Physiology \& Behavior, 23, 1109-1117.

KaPP, B. S., Gallagher, M., Underwood, M. D., McNall, C. L., \& Whitehorn, D. (1982). Cardiovascular responses elicited by electrical stimulation of the amygdala central nucleus in the rabbit. Brain Research, 234, 251-262.

KaPP, B. S., Supple, W. F. J., \& Whalen, P. J. (1994). Effects of electrical stimulation of the amygdaloid central nucleus on neocortical arousal in rabbit. Behavioral Neuroscience, 108, 81-93.

Kapp, B. S., Whalen, P. J., Supple, W. F., \& Pascoe, J. P. (1992). Amygdaloid contributions to conditioned arous $1 \mathrm{l}$ and sensory information processing. In J. P. Aggleton (Ed.), The amygdala: Neurobiological aspects of emotion, memory, and mental dysfunction (pp. 229-254). New York: Wiley-Liss.

KEHOE, E. J. (1988). A layered network model of associative learning: Learning to learn and configuration. Psychological Review, 95, 411 433.

KeHOE, E. J. (1990). Classical conditioning: Fundamental issues for adaptive network models. In M. Gabriel \& J. Moore (Eds.), Learning and computational neuroscience: Foundations of adaptive networks (pp. 389-420). Cambridge, MA: MIT Press.

KeHOE, E. J., Horne, P. S., MACrae, M., \& Horne, A. J. (1993). Realtime processing of the serial stimuli in classical conditioning of the rabbit's nictitating membrane response. Journal of Experimental Psychology: Animal Behavior Processes, 19, 265-283.

Kelso, S. R., \& Brown, T. H. (1986). Differential conditioning of associative synaptic enhancement in hippocampal brain slices. Science, 232, 85-87.

Kelso, S. R., Ganong, A. H., \& Brown, T. H. (1986). Hebbian synapses in hippocampus. Proceedings of the National Academy of Sciences, 83, 5326-5330.

KESNER, R. P. (1992). Learning and memory in rats with an emphasis on the role of the amygdala. In J. P. Aggleton (Ed.), The amygdala: Neurobiological aspects of emotion, memory, and mental dysfunction (pp. 379-399). New York: Wiley-Liss.

Killcross, S., Robrins, T. W., \& EveritT, B. J. (1997). Different types of fear-conditioned behaviour mediated by separate nuclei within amygdala. Nature, 388, 377-380.

Kim, J. J., KRUPA, D. J., \& Thompson, R. F. (1998). Inhibitory cerebelloolivary projections and blocking effect in classical conditioning. Science, 279, 570-573.

Kıм, J. J., \& ThOmpson, R. F. (1997). Cerebellar circuits and synaptic mechanisms involved in classical eyeblink conditioning. Trends in Neurosciences, 20, 177-181.

Kirkwood, A., \& BeAR, M. F. (1994). Hebbian synapses in visual cortex. Journal of Neuroscience, 14, 1634-1645

KIRKWOOD, A., LEE, H.-K., \& BEAR, M. F. (1995). Co-regulation of long-term potentiation and experience-dependent synaptic plasticity in visual cortex by age and experience. Nature, 375, 328-331.

KOHONEN, T. (1984). Self-organization and associative memory. New York: Springer-Verlag.

Krettek, J. E., \& Price, J. L. (1978). A description of the amygdaloid complex in the rat and cat with observations on intra-amygdaloid axonal connections. Journal of Comparative Neurology, 178, 255-280.

LAM, Y.-W., Wong, A., CANLI, T., \& Brown, T. H. (1996). Conditioned enhancement of the early component of the rat eyeblink reflex. Neurobiology of Learning \& Memory, 66, 212-220.
Lavond, D. G., Kim, J. J., \& Thompson, R. F. (1993). Mammalian brain substrates of aversive classical conditioning. Annual Review of Psychology, 44, 317-342.

LEDouX, J. E. (1990). Information flow from sensation to emotion Plasticity in the neural computation of stimulus value. In M. Gabriel \& J. Moore (Eds.), Learning and computational neuroscience: Foundations of adaptive networks (pp. 3-51). Cambridge, MA: MIT Press.

LEDoux, J. E. (1993). Emotional memory systems in the brain. Behavioural Brain Research, 58, 69-79.

LEDoux, J. E. (1995). Emotion: Clues from the brain. Annual Review of Psychology, 46, 209-235.

LeDoux, J. E., Cicchetti, P., Xagoraris, A., \& Romanski, L. M. (1990). The lateral amygdaloid nucleus: Sensory interface of the amygdala in fear conditioning. Journal of Neuroscience, 10, 10621069.

Le Gal La Salle, G., \& BeN-Ari, Y. (1981). Unit activity in the amygdaloid complex: A review. In Y. Ben-Ari (Ed.), The amygdaloid complex (pp. 227-237). New York: Elsevier, North-Holland.

LEVY, W. B., \& STEWARD, O. (1979). Synapses as associative memory elements in the hippocampal formation. Brain Research, 175, 233245

Lopez-Barneo, J., Hoshi, T., Heinemann, S. H., \& Aldrich, R. W. (1993). Effects of external cations and mutations in the pore region on C-type inactivation of Shaker potassium channels. Receptors \& Channels, 1, 61-71.

MAGeE, J. C., \& Johnston, D. (1997). A synaptically controlled, associative signal for Hebbian plasticity in hippocampal neurons. Science, 275, 209-213.

MAREN, S. (1996). Synaptic transmission and plasticity in the amygdala. Molecular Neurobiology, 13, 1-22.

Mascagni, F., McDonald, A. J., \& Coleman, J. R. (1993). Corticoamygdaloid and corticocortical projections of the rat temporal cortex: A Phaseolus vulgaris leucoagglutinin study. Neuroscience, 57, 697-715.

Mauk, M. D., \& Donegan, N. H. (1997). A model of Pavlovian eyelid conditioning based on the synaptic organization of the cerebellum. Learning \& Memory, 4, 130-158.

MCDOnaLD, A. J., \& J ACKSON, T. R. (1987). Amygdaloid connections with posterior insular and temporal cortical areas in the rat. Journal of Comparative Neurology, 262, 59-77.

Mial, C. (1989). The storage of time intervals using oscillating neurons. Neural Computation, 1, 359-371.

Miller, M. W., \& VoGT, B. A. (1984). Direct connections of rat visual cortex with sensory, motor, and association cortices. Journal of Comparative Neurology, 226, 184-202.

Moore, J. W., Berthier, N. E., \& Blazis, D. E. (1990). Classical eyeblink conditioning: Brain systems and implementation of a computational model. In M. Gabriel \& J. Moore (Eds.), Learning and computational neuroscience: Foundations of adaptive networks (pp. 359-387) Cambridge, MA: MIT Press.

MoORE, J. W., \& ChOI, J.-S. (1997). Conditioned response timing and integration in the cerebellum. Learning \& Memory, 4, 116-129.

Moore, J. W., Choi, J.-S., \& Brunzell, D. H. (1998). Predictive timing under temporal uncertainty: The time derivative model of the conditioned response. In D. A. Rosenbaum \& C. E. Collyer (Eds.), Timing of behavior: Neural, psychological, and computational perspectives (pp. 3-34). Cambridge, MA: MIT Press.

Moore, J. W., Desmond, J. E., \& Berthier, N. E. (1989). Adaptively timed conditioned responses and the cerebellum: A neural network approach. Biological Cybernetics, 62, 17-28

Mulkey, R. M., Herron, C. E., \& Malenka, R. C. (1993). An essential role for protein phosphatases in hippocampal long-term depression. Science, 261, 1051-1055.

Neveu, D., \& ZuCker, R. S. (1996). Postsynaptic levels of $\left[\mathrm{Ca}^{2+}\right]_{i}$ needed to trigger LTD and LTP. Neuron, 16, 619-629.

Nicoll, R. A., \& MalenKa, R. C. (1995). Contrasting properties of two forms of long-term synaptic potentiation in the hippocampus. Nature, 377, 115-118.

Oliet, S. H. R., Malenka, R. C., \& Nicoll, R. A. (1996). Bidirectional control of quantal size by synaptic activity in the hippocampus. Science, 271, 1294-1297.

Oliet, S. H. R., Malenka, R. C., \& Nicoll, R. A. (1997). Two distinct 
forms of long-term depression coexist in CA1 hippocampal pyramidal neurons. Neuron, 18, 969-982.

Quirk, G. J., RePA, C., \& LeDoux, J. E. (1995). Fear conditioning enhances short-latency auditory responses of lateral amygdala neurons: Parallel recordings in the freely behaving rat. Neuron, 15, 1029-1039.

RaYmond, J. L., LisBerger, S. G., \& MAUK, M. D. (1996). The cerebellum: A neuronal learning machine? Science, 272, 1126-1131.

Rescorla, R. A., Durlach, P. J., \& Grau, J. W. (1985). Contextual learning in Pavlovian conditioning. In P. D. Balsam \& A. T. Tomie (Eds.), Context and learning (pp. 23-56). Hillsdale, NJ: Erlbaum.

RogAN, M. T., \& LeDoux, J. E. (1995). LTP is accompanied by commensurate enhancement of auditory-evoked responses in a fear conditioning circuit. Neuron, 15, 127-136.

Romanski, L. M., Clugnet, M.-C., Bordi, F., \& LeDoux, J. E. (1993). Somatosensory and auditory convergence in the lateral nucleus of the amygdala. Behavioral Neuroscience, 107, 444-450.

RoMANSKI, L. M., \& LeDouX, J. E. (1992). Equipotentiality of thalamoamygdala and thalamo-cortico-amygdala circuits in auditory fear conditioning. Journal of Neuroscience, 12, 4501-4509.

Romanski, L. M., \& LeDoux, J. E. (1993). Information cascade from primary auditory cortex to the amygdala: Corticocortical and corticoamygdaloid projections of temporal cortex in the rat. Cerebral Cortex, 3, 515-532.

Rosen, J. B., Hitchcock, J. M., Miserendino, M. J., Falls, W. A., CAmpeau, S., \& Davis, M. (1992). Lesions of the perirhinal cortex but not of the frontal, medial prefrontal, visual, or insular cortex block fear-potentiated startle using a visual conditioned stimulus. Journal of Neuroscience, 12, 4624-4633.

Rosenbaum, D. A., \& Collyer, C. E. (Eds.) (1998). Timing of behavior: Neural, psychological, and computational perspectives. Cambridge, MA: MIT Press.

Schmajuk, N. A. (1997). Animal learning and cognition: A neural network approach. Cambridge, MA: Cambridge University Press.

Scott, S. K., Young, A. W., Calder, A. J., Hellawell, D. J., AGGLETON, J. P., \& JOHNSON, M. (1997). Impaired auditory recognition of fear and anger following bilateral amygdala lesions. Nature, 385, 254-257.

SHORS, T. J., \& MATZEL, L. D. (1997). Long-term potentiation: What's learning got to do with it? Behavioral \& Brain Sciences, 20, 597-655.

Stefanacci, L., Farb, C. R., Pitkänen, A., Go, G., LeDoux, J. E., \& Amaral, D. G. (1992). Projections from the lateral nucleus to the basal nucleus of the amygdala: A light and electron microscopic PHA-L study in the rat. Journal of Comparative Neurology, 323, 586-601.

Stevens, C. F. (1996). Strengths and weaknesses in memory. Nature, $381,471-472$.

SteVens, C. F., \& WANG, Y. (1994). Changes in reliability of synaptic function as a mechanism for plasticity. Nature, 371, 704-707.

Sullivan, W. E. (1982). Possible neural mechanisms of target distance coding in auditory system of the echolocating bat Myotis lucifugus. Journal of Neurophysiology, 48, 1033-1047.

Sutton, R. S., \& Barto, A. G. (1981). Toward a modern theory of adaptive networks: Expectation and prediction. Psychological Review, 88, 135-170.

Sutton, R. S., \& Barto, A. G. (1990). Time-derivative models of
Pavlovian reinforcement. In M. Gabriel \& J. Moore (Eds.), Learning and computational neuroscience: Foundations of adaptive networks (pp. 497-537). Cambridge, MA: MIT Press.

Swanson, L. W., \& Petrovich, G. D. (1998). What is the amygdala? Trends in Neurosciences, 21, 323-331.

Teyler, T. J., Cavus, I., Coussens, C., DiScenna, P., Grover, L., Lee, Y. P., \& LiTTLE, Z. (1994). Multideterminant role of calcium in hippocampal synaptic plasticity. Hippocampus, 4, 623-634.

THOMPSON, R. F. (1986). The neurobiology of learning and memory. Science, 233, 941-947.

Thompson, R. F., \& Krupa, D. J. (1994). Organization of memory traces in the mammalian brain. Annual Review of Neuroscience, 17, 519-549.

Tieu, K. H., Faulkner, B., \& Brown, T. H. (1996). Perirhinal-amygdala neural network model of temporal encoding in fear conditioning. Society for Neuroscience Abstracts, 22, 1742.

Treisman, M., Cook, N., Naish, P. L. N., \& MacCrone, J. K. (1994). The internal clock: Electroencephalographic evidence for oscillatory processes underlying time perception. Quarterly Journal of Experimental Psychology, 47A, 241-289.

TURNER, B. H., \& ZimMER, J. (1984). The architecture and some of the interconnections of the rat's amygdala and lateral periallocortex. Journal of Comparative Neurology, 227, 540-557.

Wallenstein, G. V., Eichenbaum, H., \& Hasselmo, M. E. (1998). The hippocampus as an associator of discontiguous events. Trends in Neurosciences, 21, 317-323.

WEBER, E. H. (1851). Annotationes anatomicae et physiologicae [Anatomical and physiological annotations]. Leipzig: C. F. Koehler. Whalen, P. J., \& KAPP, B. S. (1991). Contributions of the amygdaloid central nucleus to the modulation of the nictitating membrane reflex in the rabbit. Behavioral Neuroscience, 105, 141-153.

Xiang, Z., Greenwood, A. C., Kairiss, E. W., \& Brown, T. H. (1994). Quantal mechanism of long-term potentiation in hippocampal mossy-fiber synapses. Journal of Neurophysiology, 71, 2552-2556. Young, B. J., OtTo, T., Fox, G. D., \& EichenbauM, H. (1997). Memory representation within the parahippocampal region. Journal of Neuroscience, 17, 5183-5195.

\section{NOTES}

1. There are several recent general reviews of the overall anatomical organization of the amygdala and/or the perirhinal cortex (see Burwell \& Amaral, 1998; Burwell, Witter, \& Amaral, 1995; Faulkner, 1997; Swanson \& Petrovich, 1998).

2. The maximum possible input intensity $\left(I_{\max } \approx 98\right)$ can occur in the special case of a second layer ALa neuron receiving US input $(I=60)$, the maximum possible CS input for plastic synapses $\left(I_{\max }=28\right)$, and high noise $\left(I_{\text {noise } \max \approx 10}\right)$.

3. Recall that testing trials never produce output when the CS and US are unpaired in the conditioning trials. Therefore, any and all output from the second-layer the ALa neurons reflects associative conditioning, because there is no spontaneous activity in these neurons and pseudoconditioning causes no output.

4. The acronym VET derives from "associative values based on $e x-$ pectation about $t$ iming" (Moore \& Choi, 1997).

\section{APPENDIX A}

Equations Governing Fast-Spiking Neurons

For FS cells, the accumulator, denoted $A(t)$, is updated according to the following rules:

$$
A(t):=\left\{\begin{array}{cc}
I(t) & U(t) \geq \theta_{A}, I(t)>0 \\
\theta_{A}-c_{A} & A(t-1) \geq \theta_{A}, I(t) \leq 0, \\
U(t) & \text { otherwise }
\end{array}\right.
$$

where $I(t)$ is the summed input to the neuron at time $t ; \theta_{A}$ is the neuron's spiking threshold; and $c_{A}$ is a constant. $U(t)$ is an update function, defined as follows:

$$
U(t):=A(t-1)+\frac{I(t)-A(t-1)}{\tau_{A}} \cdot \Delta t
$$


APPENDIX A (Continued)

where $\tau_{A}$ is a time constant for the accumulator; $A(t-1)$ in the numerator is a dissipation term; and $\Delta t$ is the time step ( $1 \mathrm{msec}$ ). The values of all constants used in the equations in Appendices $\mathrm{A}-\mathrm{C}$ are given in Table $\mathrm{E} 1$ of Appendix E.

When a neuron reaches its threshold for spiking, the output of the processing unit is set to 1 , indicating that the cell spiked when it crossed threshold. Following the cell's first spike, the frequency generator is activated. This module takes the value of the accumulator, $A(t)$, and computes a spiking frequency (in hertz), $\varphi(t)$, according to the sigmoid function:

$$
\varphi(t):=\left\{\begin{array}{cc}
\frac{\varphi_{\max }-\varphi_{\min }}{1+\exp \left(-\frac{A(t)-c_{\delta}}{c_{\varphi}}\right)}+\varphi_{\min } & A(t) \geq \theta_{A} \\
0 & \text { otherwise }
\end{array},\right.
$$

where $\varphi_{\max }$ and $\varphi_{\min }$ are, respectively, the maximum and minimum spiking frequencies, $c_{\varphi}$ is a constant that sets the slope of this sigmoid, and $c_{\delta}$ is a constant that shifts the dynamic range of the function relative to its inputs. Equation 3 produces a sigmoid input-output function and allows for a minimum firing frequency $\varphi_{\min }$. In the simulations shown here, $\varphi_{\min }$ is zero (Table El of Appendix E). The spike generator takes the spiking frequency $\varphi(t)$ and sets the output of the processing unit $O(t)$ to either a 1 or a 0 , according to the equation

$$
O(t):=\left\{\begin{array}{cc}
1 & \left(t-t_{\mathrm{ps}}\right) \geq[\text { int }] \\
0 & \begin{array}{c}
1,000 \mathrm{msec} \\
\text { otherwise }
\end{array}, A(t) \geq \theta_{A}
\end{array},\right.
$$

where [int] indicates that fractional values are truncated before applying the test, and $t_{\mathrm{ps}}$ is the time of the previous spike (recall that the neuron always spikes when the threshold is crossed for the first time, before $t_{\mathrm{ps}}$ has a legitimate value). The remaining state variables are then updated as follows:

$$
\begin{gathered}
I(t):=0 ; \\
t_{p s}:=\left\{\begin{array}{cc}
t & O(t)=1 \\
t_{p s} & \text { otherwise }
\end{array}\right.
\end{gathered}
$$

\section{APPENDIX B \\ Equations Governing Regular-Spiking Neurons}

The frequency and spike generators are the same in RS cells as in FS cells, except for the value of $c_{\delta}$ (Equation 3), which is slightly greater for RS cells (Table El of Appendix E). The major difference between the two cell types is that RS cells contain a fifth module in their implementation, called the accommodator (see Figure 2), which affects the recurrence relation governing the accumulator. As the value of the accumulator approaches spiking threshold, $A(t)$ is given by the following equation:

$$
A(t):=A(t-1)+\frac{I(t)-A(t-1)-K(t)}{\tau_{A}} \cdot \Delta t,
$$

where $K(t)$ is an accommodation term produced by the accommodator module. When the unit reaches the threshold for spiking, $\theta_{A}$, the value of the accumulator is increased to equal the current input:

$$
A(t):=I(t),
$$

and the processing unit's output, $O(t)$, is set to 1 , indicating that the cell spiked when it crossed threshold.

Once the cell exceeds threshold, the value of its accumulator is governed by the equation:

$$
A(t):=\left\{\begin{array}{cc}
\theta_{\mathrm{A}}-c_{\mathrm{A}} & I(t) \leq 0 \\
A(t-1)+\frac{I(t)-A(t-1)-K(t)}{\tau_{A}} \cdot \Delta t & \text { otherwise }
\end{array}\right.
$$

However, Equation 7 replaces Equation 9 whenever $A(t)$ drops below spiking threshold. $K(t)$ and the terms on which it depends are defined as follows: 


$$
\begin{gathered}
K(t):=\left\{\begin{array}{cc}
n(t)^{4} \cdot K_{\max } & I(t)>0 \\
0 & \text { otherwise }
\end{array}\right. \\
n(t):=n(t-1)+\frac{n_{\infty}(A)-n(t-1)}{\tau_{n}(A)} \cdot \Delta t ;
\end{gathered}
$$

$K_{\text {max }}$ is a positive constant, and $n_{\infty}(A)$ and $\tau_{n}(A)$ are given by:

$$
\begin{gathered}
n_{\infty}(A):=\frac{1}{1+\exp \left[-\left(A(t)-c_{n_{\infty}}\right)\right]} \\
\tau_{n}(A):=\frac{\tau_{n, \max }}{1+\exp \left(-\frac{A(t)-c_{1 \tau_{n}}}{c_{2 \tau_{n}}}\right)}+c_{\tau_{n}, \min } .
\end{gathered}
$$

Accommodation is modeled by $K(t)$, which can be likened to a potassium conductance governed by a maximum value $K_{\max }$ and an activation term $n(t)$, which exponentially approaches $n_{\infty}(A)$, the limiting value of $n(t)$ for a given magnitude of $A$. An additional function, denoted $S(t)$, secures full accommodation in RS cells. $S(t)$ is initially zero, in which case it has no effect on the RS cell. During a learning trial, $S(t)$ monitors the time that has elapsed since the neuron's most recent spike $\left(t-t_{\mathrm{ps}}\right)$. If this amount of time exceeds a predetermined multiple $(\lambda)$ of the initial interspike interval $\left(\mathrm{ISpI}_{0}\right)$, and if two additional conditions are also met (one of which specifies that $\mathrm{ISpI}_{0}$ must exist), then $S(t)$ is set to one:

$$
S(t):=\left\{\begin{array}{lc}
1 & \mathrm{ISpI}_{0}>0, t-t_{\mathrm{ps}}>\lambda \cdot \mathrm{ISpI}_{0}, A(t)<\theta_{\mathrm{A}} \\
0 & \text { otherwise }
\end{array} .\right.
$$

If $S(t)$ becomes equal to one, the parameters held in the state are immediately changed as follows:

$$
\begin{aligned}
& \varphi(t):=0 ; \\
& O(t):=0 .
\end{aligned}
$$

The ultimate effect of Equations 14-16 is that once $S(t)$ becomes 1, the neuron will not spike for the remainder of the trial (recall that there are six trials in each conditioning session). $S(t)$ ensured accommodation in all RS cells except for those in the second layer of the ALa, which often receive temporally disjoint inputs produced by the CS and US and therefore may emit more than one train of spikes. Recall that our model neurons cannot fire in the absence of input. Whenever $S(t)$ causes a RS neuron to stop firing, all the subsequent neurons in that chain cease firing as well (except for the terminal neuron, which can be fired by the US). Synaptic modification does not occur if either the pre- or the postsynaptic neuron has a spiking frequency of zero.

Once any neuron in a chain stops firing, no further synaptic changes can occur in that chain until the next trial. Note that all the chains (both LS and RS) are terminated by two RS neurons in the ALa and that, once the first of these (the preterminal neuron) stops firing, no turther LTP or LTD can occur within that chain for the remainder of the trial. The run times were reduced significantly by setting $S(t)$ equal to 1 for all nonterminal neurons in a chain once $S(t)$ became 1 for any nonterminal neuron in that chain. 


\section{APPENDIX C \\ Equations Governing Late-Spiking Neurons}

LS cells are represented by equations similar to those used for RS cells, with two exceptions. First, when a LS cell crosses threshold, the value of its accumulator, $A(t)$, is set to the value

$$
A(t):=A(t-1)+c_{1_{1}} \cdot I(t)^{2}-c_{1_{2}} \cdot I(t)+c_{1_{3}},
$$

where $c_{1}, c_{12}$, and $c_{1_{3}}$ are constants (Equation 17 replaces Equation 7). Second, the accommodation term, $K(t)$, is redefined and also includes "inactivation," $h(t)$, such that

$$
\begin{gathered}
K(t):=\left\{\begin{array}{cr}
n(A)^{4} \cdot h(t) \cdot K_{\max } & I(t)>0 \\
0 & \text { otherwise }
\end{array}\right. \\
h(t):=h(t-1)+\frac{h_{\infty}(A)-h(t-1)}{\tau_{h}(A)} \cdot \Delta t ; \\
h_{\infty}(A):=\frac{1}{1+\exp \left(A(t)-c_{h_{\infty}}\right)},
\end{gathered}
$$

where $h_{\infty}(A)$ is the asymptotic value of $h(t)$ for any given value of $A(t)$. The terms $n(A)$ and $\tau_{h}(A)$ are defined as follows:

$$
\begin{gathered}
n(A):=\frac{1}{1+\exp \left[-\left(A(t)-c_{n}\right)\right]} ; \\
\tau_{h}(A):=\frac{\tau_{h, \max }}{1+\exp \left[\frac{A(t)-c_{\tau_{h,},}}{c_{\tau_{h}, 2}}\right]}+c_{\tau_{h}, \min }
\end{gathered}
$$

Note that Equation 21 differs from Equation 11 in that it represents an instantaneous activation as a function of the value of the accumulator $(A)$.

\section{APPENDIX D \\ Equations Governing Synapses}

The strength of the synaptic input to postsynaptic neuron $i$ from presynaptic neuron $j$ is termed a synaptic weight and is denoted $w_{i j}$. The synaptic input to postsynaptic neuron $i, I_{i}(t)$, is just the sum of the products of the weights and their corresponding presynaptic activities, $a_{j}$ :

$$
I_{i}(t):=\sum_{j} w_{i j} a_{j} .
$$

Synaptic noise is represented as follows:

$$
I_{i}\left(t, z, \kappa_{c v}\right):=\left(1+\kappa_{c v} z\right) \sum_{j} w_{i j} a_{j},
$$

where $z$ is a standardized Gaussian variate (mean of zero and standard deviation of unity) and $\kappa_{c v}$ is a random variable that sets the coefficient of variation of the noise modulation of the input. $\boldsymbol{\kappa}_{c v}$ is drawn from a uniform distribution with limits of 0.000 and 0.100 .

At plastic synapses, the weight at time $t$ between neurons $i$ and $j$ is updated on each subsequent time step according to the recurrence relation:

$$
w_{i j}(t):=\left\{\begin{array}{cc}
w_{i j}(t-1)+\Delta w_{i j}(t-1) & L<w_{i j}(t-1)+\Delta w_{i j}(t-1)<U \\
L & w_{i j}(t-1)+\Delta w_{i j}(t-1) \leq L \\
U & w_{i j}(t-1)+\Delta w_{i j}(t-1) \geq U
\end{array},\right.
$$




\section{APPENDIX D (Continued)}

where $\Delta w_{i j}(t)$ is the change in the strength of the connection between neurons $i$ and $j$ at time $t$, and $L$ and $U$ are the lower and upper bounds on $w_{i j}(t)$, respectively. The values of all the constants used in the equations governing plastic synapses are listed in Table E2 of Appendix E.

$\Delta w_{i j}(t)$ from Equation 25 is determined as follows:

$$
\Delta w_{i j}(t):=\phi\left\{a_{i}(t), \theta_{p}, \theta_{d}\right\} \cdot \eta\left\{a_{j}(t)\right\},
$$

where $a_{i}(t)$ is the postsynaptic spike frequency and $\eta\left\{a_{j}(t)\right\}$ is the differentially weighted presynaptic spike frequency. The quantity $\phi\left\{a_{i}(t), \theta_{p}, \theta_{d}\right\}$ is the postsynaptic contribution to potentiation $(P)$ or depression $(D)$ :

$$
\phi\left\{a_{i}(t), \theta_{p}, \theta_{d}\right\}:=\left\{\begin{array}{cc}
0 & a_{i}(t) \leq \theta_{d} \\
D & \theta_{d}<a_{i}(t)<\theta_{p} \\
0 & a_{i}(t)=\theta_{p} \\
P & a_{i}(t)>\theta_{p}
\end{array},\right.
$$

where $\theta_{d}$ and $\theta_{p}$ are the thresholds for depression and potentiation, respectively, and $D$ and $P$ are given by the following set of equations:

$$
\begin{gathered}
D:=\alpha \cdot a_{i}(t)^{2}+\beta \cdot a_{i}(t)+\gamma ; \\
P:=\left(2 \alpha \cdot \theta_{p}+\beta\right)\left(a_{i}(t)-\theta_{p}\right), \\
\beta:=\alpha \cdot\left(\frac{\theta_{d}{ }^{2}-\theta_{p}{ }^{2}}{\theta_{p}-\theta_{d}}\right), \\
\gamma:=\alpha \cdot \theta_{d} \cdot \theta_{p},
\end{gathered}
$$

where $\alpha$ is a constant greater than zero, $\beta<0$, and $\gamma>0$.

The expression $\eta\left\{a_{j}(t)\right\}$ in Equation 25 is defined as

$$
\eta\left\{a_{j}(t)\right\}:=\left\{\begin{array}{ll}
n_{1} \cdot a_{j}(t) & a_{i}(t)>\theta_{p} \\
n_{2} \cdot a_{j}(t) & \text { otherwise }
\end{array},\right.
$$

where $n_{1}>n_{2}$, which has the effect of increasing the rate of potentiation relative to depression, other things being equal. 
APPENDIX E

Parameters and Constants for Neurons and Synapses

Table E1

Parameters Used in Constructing RS, LS, and FS Cell Types

\begin{tabular}{|c|c|c|c|c|}
\hline Symbol & Equations & RS & LS & F S \\
\hline $\overrightarrow{\theta_{A}}$ & $1,3,9,14$ & 20 & 20 & 20 \\
\hline$c_{A}$ & 1,9 & 1 & 1 & 1 \\
\hline \multirow[t]{4}{*}{$\tau_{A}$} & $2,7,9$ & RS1 46.5 & \multirow[t]{4}{*}{46.5} & \multirow[t]{4}{*}{10} \\
\hline & & RS2 92 & & \\
\hline & & RS3 183 & & \\
\hline & & RS4 274 & & \\
\hline$\varphi_{\max }$ & 3 & 40 & 40 & 120 \\
\hline$\varphi_{\min }$ & 3 & 0 & 0 & 0 \\
\hline$c_{\delta}$ & 3 & 28 & 28 & 26 \\
\hline$c_{\varphi}$ & 3 & 1.5 & 1.5 & 1.5 \\
\hline$K_{\max }$ & 10,18 & $15 \times 10^{6}$ & 38 & NA \\
\hline$c_{n_{\infty}}$ & 12 & 20 & NA & $\mathrm{NA}$ \\
\hline$\tau_{n, \max }$ & 13 & $40 \times 10^{3}$ & NA & $\mathrm{NA}$ \\
\hline$c_{\tau_{n}, \min }$ & 13 & 5 & $\mathrm{NA}$ & NA \\
\hline$c_{1 t_{n}}$ & 13 & 20 & NA & $\mathrm{NA}$ \\
\hline$c_{2 \tau_{n}}$ & 13 & 10 & $\mathrm{NA}$ & NA \\
\hline$\lambda$ & 14 & 4 & NA & NA \\
\hline$c_{11}$ & 17 & NA & 0.0408217 & NA \\
\hline$c_{12}$ & 17 & NA & 1.46387 & $\mathrm{NA}$ \\
\hline$c_{13}$ & 17 & $\mathrm{NA}$ & 12.4152 & $\mathrm{NA}$ \\
\hline$c_{h_{\infty}}$ & 20 & NA & 15 & NA \\
\hline$c_{n}^{\infty}$ & 21 & NA & 15 & NA \\
\hline$\tau_{h, \max }^{\prime \prime}$ & 22 & NA & $(\mathrm{LS} 1 \quad 400$ & NA \\
\hline & & & LS2 745 & \\
\hline & & & LS3 1622 & \\
\hline & & & LS4 5150 & \\
\hline$c_{\tau_{h, \min }}$ & 22 & NA & 5 & NA \\
\hline$c_{t_{h}, 1}$ & 22 & $\mathrm{NA}$ & 15 & $\mathrm{NA}$ \\
\hline$c_{\tau_{h}, 2}$ & 22 & NA & 4 & $\mathrm{NA}$ \\
\hline
\end{tabular}

Table E2

Parameters Used in Computing Synaptic Plasticity

\begin{tabular}{cll}
\hline Symbol & \multicolumn{1}{c}{ Equations } & Value \\
\hline$L$ & 25 & 0 \\
$U$ & 25 & 28 \\
$\theta_{p}$ & $26,27,30-32$ & 38.7 \\
$\theta_{d}$ & $26,27,30,31$ & 0.5 \\
$\alpha$ & $28-31$ & 1.0 \\
$n_{1}$ & 32 & $6 \times 10^{-5}$ \\
$n_{2}$ & 32 & $1 \times 10^{-5}$ \\
\hline
\end{tabular}

(Manuscript received October 6, 1997;

revision accepted for publication October 19, 1998.) 